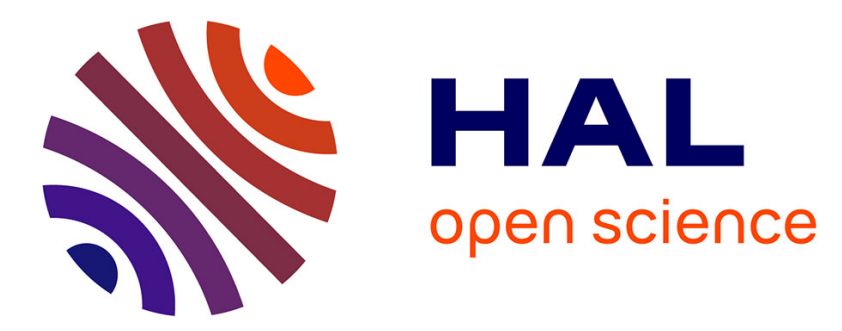

\title{
Paramagnetic relaxation effects in perturbed angular correlations for arbitrary electronic relaxation time
}

\author{
C. Chopin, D. Spanjaard, F. Hartmann-Boutron
}

\section{To cite this version:}

C. Chopin, D. Spanjaard, F. Hartmann-Boutron. Paramagnetic relaxation effects in perturbed angular correlations for arbitrary electronic relaxation time. Journal de Physique, 1975, 36 (10), pp.961-978. 10.1051/jphys:019750036010096100 . jpa-00208341

\section{HAL Id: jpa-00208341 \\ https://hal.science/jpa-00208341}

Submitted on 1 Jan 1975

HAL is a multi-disciplinary open access archive for the deposit and dissemination of scientific research documents, whether they are published or not. The documents may come from teaching and research institutions in France or abroad, or from public or private research centers.
L'archive ouverte pluridisciplinaire HAL, est destinée au dépôt et à la diffusion de documents scientifiques de niveau recherche, publiés ou non, émanant des établissements d'enseignement et de recherche français ou étrangers, des laboratoires publics ou privés. 


\title{
PARAMAGNETIC RELAXATION EFFECTS IN PERTURBED ANGULAR CORRELATIONS FOR ARBITRARY ELECTRONIC RELAXATION TIME
}

\author{
C. CHOPIN, D. SPANJAARD, F. HARTMANN-BOUTRON
}

Laboratoire de Physique des Solides (*), Université Paris-Sud, 91405 Orsay, France

(Reçu le 5 mars 1975, accepté le 9 mai 1975)

\begin{abstract}
Résumé. - Les traitements de perturbation utilisés jusqu'à présent pour calculer les effets de la relaxation paramagnétique dans les expériences de corrélations angulaires perturbées $\gamma \gamma$ n'étaient valables que pour des temps de relaxation électronique très courts. Nous tournons cette difficulté en adaptant à ce problème la nouvelle théorie de perturbations mise au point récemment par Hirst et d'autres auteurs afin d'étudier les effets de relaxation dans les spectres Mössbauer. Moyennant l'hypothèse de relaxation électronique sphérique, il devient alors possible de calculer les facteurs de perturbation en fonction de paramètres de relaxation qui sont directement reliés au hamiltonien de relaxation microscopique. Nous comparons nos résultats à ceux de la théorie stochastique de Scherer et Blume.
\end{abstract}

\begin{abstract}
Previous perturbation treatments of paramagnetic relaxation effects in $\gamma \gamma$ PAC were limited to the case of very short electronic relaxation times. We circumvent this limitation by invoking a new perturbation theory recently elaborated by Hirst and others for handling relaxation effects in Mössbauer spectra. Under the assumption of spherical electronic relaxation we are then able to compute the perturbation factors as functions of certain relaxation parameters which are directly related to the microscopic relaxation hamiltonian. We compare our results to those of the stochastic theory of Scherer and Blume.
\end{abstract}

1. Introduction ( $\left.{ }^{1}\right)$. - Previous perturbation treatments [1-3] of paramagnetic relaxation effects in PAC were limited to the case of very short electronic relaxation times $T_{1 \mathrm{~s}}$. The aim of this paper is to show that the relaxation theory devised by Hirst and others for Mössbauer spectra [4-6], allows one to compute the perturbation factors $G_{k k^{\prime}}^{q q^{\prime}}(t)$ for a $T_{1 \mathrm{~s}}$ of arbitrary size.

Let us consider a paramagnetic impurity in a solid with a radioactive cascade $I_{\mathrm{i}} \stackrel{\mathbf{k}_{1}}{\rightarrow} I \stackrel{\mathbf{k}_{2}}{\rightarrow} I_{\mathrm{f}}$ and assume that the intermediate state $I$ observed in PAC exhibits a hyperfine interaction : $\mathscr{H}_{\mathrm{hf}}=A \mathbf{I}$.S, where $\mathrm{S}$ is the effective spin of the lowest electronic level of the impurity. Coupling of $\mathbf{S}$ with the lattice gives rise to an electronic relaxation characterized by $T_{1 \mathrm{~s}}$.

The expressions for the perturbation factors in the

(*) Associé au C.N.R.S.

$(* *)$ A preliminary account of the present work has already been published in C. Chopin, D. Spanjaard, F. Hartmann-Boutron, Compt. Rend. 278 (1974) 739. It was also the object of a short communication to the Uppsala Conference on Hyperfine Interactions (June 1974). In the figure caption of both papers one should read time unit : $T=2 h / 5 \mathrm{~A}$.

(') In this introduction, we do not intend to mention all previous studies in this field, but only those papers directly related to the present work. Further references can be found in references [2, 3, $5,7,9,10,11]$. absence of relaxation are well known and correspond to oscillating behaviour at the hyperfine frequencies [7]. On the contrary when $T_{1 \mathrm{~s}}$ is very short, the relaxation gives rise to a pure attenuation of the correlation which was computed by Abragam and Pound [1]. In order to obtain this result they assumed that the effect of relaxation is to confer to the hyperfine hamiltonian a fluctuating character :

$$
A \mathbf{I} . \mathbf{S} \rightarrow A \mathbf{I} . \mathbf{S}(t) \equiv \mathcal{H}_{1}^{\prime}(t) .
$$

This hamiltonian, whose correlation time $\tau_{c}^{\prime}$ is clearly equal to $T_{1 \mathrm{~s}}$, is then treated by a perturbation method similar to that used in chapitre 8 of Abragam's book [8]. The condition for the validity of the perturbation method is that $\left(\left\langle\mathcal{H}_{1}^{\prime 2}\right\rangle \tau_{\mathrm{c}}^{\prime 2} / \hbar^{2}\right) \ll 1$. Consequently the treatment of Abragam and Pound is limited to very short relaxation times : $(A / \hbar) T_{1 \mathrm{~s}} \ll 1$.

The intermediate case $(A / \hbar) T_{1 \mathrm{~s}} \sim 1$ was recently investigated by Scherer [9] and Blume [10] with the help of a stochastic model in which the lattice, represented by a stochastic hamiltonian $\mathcal{H}_{1}$ acting on $\mathbf{S}$, induces transitions between the eigenstates of the coupled electronuclear system (I, S). In this approach one has therefore a static hamiltonian :

$$
\mathscr{H}_{0}=\mathscr{H}_{\mathrm{hf}}=A \mathbf{I} . \mathbf{S}
$$


and a fluctuating hamiltonian corresponding to random independent pulses acting only on $\mathbf{S}$ :

$$
\mathcal{H}_{1}=\sum_{i} V_{i} \delta\left(t-t_{i}\right)
$$

As discussed by Clauser and Blume [11], the average effect of one of these pulses can be characterized by a transition superoperator $\tau_{\mathrm{av}}$ (in the Liouville representation) :

$$
\tau_{\mathrm{av}}=\left(\mathrm{e}^{i V_{i}^{\times}}\right)_{\mathrm{av}} \cdot
$$

Scherer and Blume assume that the effect of each pulse is to make the direction of the electronic spin $\mathbf{S}$ random and, as mentioned by Blume ([10] p. 83), this is equivalent to assuming that with respect to eigenstates $m_{S}, m_{S}^{\prime}$ of $S_{z}$ :

$$
\left\langle m_{S}, m_{S}^{\prime}\left|\tau_{\mathrm{av}}\right| m_{S}^{\prime \prime}, m_{S}^{\prime \prime \prime}\right\rangle=\frac{\delta_{m_{S} m_{S}^{\prime}} \delta_{m_{S}^{\prime \prime} m_{S}^{\prime \prime \prime}}}{(2 S+1)} .
$$

With these hypotheses the problem is clearly isotropic. Hence :

$$
G_{k k^{\prime}}^{q q^{\prime}}(t)=\delta_{k k^{\prime}} \delta_{q q^{\prime}} G_{k k}^{00}(t)=\delta_{k k^{\prime}} \delta_{q q^{\prime}} G_{k}(t) .
$$

On the other hand, using the explicit form of $\tau_{\mathrm{av}}$, it is possible to establish a simple relationship between the Laplace transforms $\widetilde{G}_{k}(p)$ and $\widetilde{G}_{k}^{0}(p)$ of the perturbation factors $G_{k}(t)$ and $G_{k}^{0}(t)$ in the presence or in the absence of relaxation. The quantity $\lambda$ being the average frequency of the pulses, assumed to obey a Poisson statistics, Blume demonstrates that (eq. 8 of [10]) :

$$
\tilde{G}_{k}(p)=\frac{\tilde{G}_{k}^{0}(p+\lambda)}{1-\lambda \tilde{G}_{k}^{0}(p+\lambda)}
$$

$G_{k}(t)$ is then easily obtained by inverse Laplace transformation of $\widetilde{G}_{k}(p)$. Numerical examples show that, as expected, when $\lambda$ varies from zero to infinity, $G_{k}(t)$ goes from a purely oscillating behaviour, to a damped oscillating behaviour, then to a purely damped behaviour and becomes finally equal to 1 when $\lambda=\infty$. This evolution is qualitatively very satisfying. However the parameter $\lambda$ has no clear relationship with real microscopic phenomena taking place in a solid, which are due to stationary spinlattice couplings and not to random pulses.

Finally a perturbation theory has been developped by Hirst and others [4-6] for handling relaxation effects in Mössbauer spectra. It relies on a physical analysis which is somewhat similar to that of Scherer Blume, i.e. there is also a main hamiltonian $\mathscr{H}_{0}=A \mathbf{I} . \mathbf{S}$ and a relaxation hamiltonian $\mathcal{H}_{1}$ which describes the coupling of $\mathbf{S}$ with the lattice, $\mathcal{H}_{1}$ is a microscopic stationary hamiltonian which can be written in explicit form when the relaxation mechanism is known [12]. $\mathscr{H}_{1}$ is treated by the perturbation method (Abragam [8] Chap. 8) but contrary to the Abragam-Pound theory, the correlation time $\tau_{\mathrm{c}}$ associated with $\mathscr{H}_{1}$ is now a lattice correlation time, which is always very short $\left(10^{-13}-10^{-15} \mathrm{~s}\right)$ so that the criterium :

$$
\left(\left\langle\mathscr{H}_{1}^{2}\right\rangle / \hbar^{2}\right) \tau_{\mathrm{c}}^{2} \ll 1
$$

is always satisfied. It then becomes possible to treat the intermediate region $\frac{A}{\hbar} T_{1 \mathrm{~s}} \sim 1$, provided that one abandons the secular approximation used in most relaxation theories. Hirst [4] considered a spin-lattice coupling of the form $\mathscr{H}_{1}=-2 J \mathbf{S} . \mathbf{s}$ (relaxation by the conduction electrons). In the present paper we shall consider more general relaxation mechanisms. On the other hand, in order to lower the dimensions of the differential systems or matrices which come into play, we shall generally assume that the relaxation has a spherical character [3]; this makes it possible to use tensor operator methods which have already proved to be very powerful in such problems [3], [13, 14].

As already mentioned, the perturbation approach of Hirst has the advantage of being directly related to the microscopic processes in a solid. In the case of the Mössbauer effect, it has made it possible to extract very fundamental and interesting information from the low temperature Mössbauer spectra of $\mathrm{Yb}^{170} \mathrm{Au}[5],[15,16]$. Since PAC have wider applicability than the Mössbauer effect, one may hope that the extension of Hirst's theory to this case will enable one to obtain similar information on a greater number of paramagnetic radioactive impurities.

2. Application of Hirst's theory to PAC. General formulae. - In this section we first recall some previous results relative to the relaxation of a bare nuclear spin under the effect of its coupling with the lattice, and we indicate how these results can be transposed to the relaxation of a bare electronic spin. We then consider the case of two coupled nuclear and electronic spins in the presence of electronic relaxation. We introduce various tensorial bases adapted to this problem and we derive the corresponding general expressions of the perturbation factors, which we relate to the temporal evolution of the density matrix. Finally we establish the equation of motion of the density matrix and we use it to obtain the fundamental expressions which will be used in sections 3 and 4 to compute numerically the perturbation factors both in the absence and in the presence of an external magnetic field. We also compare our results with other theories.

2.1 Summary OF PREVIOUS ReSUltS. - In reference [3] we considered the relaxation of a nuclear spin I coupled directly to the lattice by fluctuating interactions having spherical symmetry. By this we mean that 1) the spectral densities of the lattice correlation functions which appear in the relaxation equations do not depend on the frequency (white spectrum approximation) and that 2) the instantaneous fluctuations of the lattice are isotropic. The assumption of 
spherical symmetry requires that the temperature $k_{\mathrm{B}} T$ be big compared to the hyperfine hamiltonian, a condition which is always fulfilled in standard PAC experiments; on the other hand one must be aware that some relaxation processes might not fulfill the conditions for spherical relaxation.

In the case where they are fulfilled, it is interesting to use a multipole expansion of the density matrix of the nuclear spin :

$$
\sigma=\sum_{k q} \sigma_{k q}^{*} T_{k}^{q}(I) \quad(k=0, \ldots 2 I ; q=-k, \ldots,+k)
$$

with :

$$
T_{k}^{q}(I)=\sum_{m m^{\prime}} \frac{\sqrt{2 k+1}}{\sqrt{2 I+1}}\left\langle\operatorname{Imkq} \mid I k I m^{\prime}\right\rangle\left|I m^{\prime}\right\rangle\langle\operatorname{Im}| .
$$

It then appears that the relaxation equations of the various $\sigma_{k q}^{*}$ are completely decoupled :

$$
\frac{\mathrm{d} \sigma_{k q}^{*}}{\mathrm{~d} t}=-\mu_{k}^{q} \sigma_{k q}^{*}=-\mu_{k} \sigma_{k q}^{*}
$$

and that when relaxation is due to a tensorial interaction of order $l\left(H_{1}=\sum_{p} R_{p}^{l}(I) F^{p}\right.$ with $\left.l \leqslant 2 I\right)$, the relaxation constants $\mu_{k} \equiv{ }^{l} \mu_{k}$ are given as functions of a unique parameter $J_{l}$ by ([3] eq. (29))

$$
{ }^{l} \mu_{k}=\frac{J_{l}}{\hbar^{2}} \frac{\left|\left\langle I\left\|R_{l}\right\| I\right\rangle\right|^{2}}{2 I+1}(1-(2 I+1) W(I l k I \mid I I))
$$

where $W$ is a Racah symbol ( $\simeq 6 j$ coefficient $)$ and :

$$
J_{l}=\int_{-\infty}^{+\infty} \frac{\overline{F^{0}(0) F^{0}(-\tau)+F^{0}(-\tau) F^{0}(0)}}{2} \mathrm{~d} \tau
$$

(the bar denotes an average over the lattice). In the case when several interactions with different tensorial orders are acting on $\mathbf{I}$, and assuming that they are statistically independent, we have :

$$
\mu_{k}=\sum_{l}^{l} \mu_{k} \quad(l \leqslant 2 I) .
$$

On the other hand we have demonstrated that (13) :

$$
\sigma_{k q}^{*}(t)=\sum_{k^{\prime} q^{\prime}} G_{k^{\prime} k}^{q^{\prime} q}\left(t, t^{\prime}\right) \sigma_{k^{\prime} q^{\prime}}^{*}\left(t^{\prime}\right)
$$

where the $G$ are the perturbation factors which can be observed in a PAC experiment on a cascade with $I$ as its intermediate state. Consequently the $G$ can be easily obtained by integration of the equation of motion of the $\sigma_{k}^{q}$ and in the simple case eq. (9) we immediately get

$$
G_{k k^{\prime}}^{q q^{\prime}}(t)=\delta_{k k^{\prime}} \delta_{q q^{\prime}} \exp \left(-\mu_{k}^{q} t\right)
$$

In addition to eq. (13), one can also establish the general relation [13] :

$G_{k^{\prime} k}^{q^{\prime} q}\left(t, t^{\prime}\right)=\operatorname{Trace}_{I}\left\{T_{k}^{q+}(I) \Lambda\left(t, t^{\prime}\right) T_{k^{\prime}}^{q^{\prime}}(I) \Lambda^{+}\left(t, t^{\prime}\right)\right\}$

where $\Lambda$ is the evolution operator in state $I$.

In the present paper we shall have to deal with a much more complicated situation than the preceding one, i.e. two coupled spins $\mathbf{I}$ and $\mathbf{S}$ with relaxation acting this time on $\mathbf{S}$ alone $\left({ }^{2}\right)$. In order to simplify it we shall assume that, in the absence of nuclear spin, the relaxation of $\mathbf{S}$ has spherical symmetry. We can thus transpose to $\mathbf{S}$ the results which were previously derived for I ; i.e., if we perform a multipole expansion of the density matrix of $\mathbf{S}$ as

$$
\sigma^{\prime}=\sum_{k q} \sigma_{k q}^{* *} T_{k}^{q}(S)
$$

then in the presence of a spin lattice coupling

$$
\sum_{l p} R_{l}^{p}(S) F^{p}
$$

we have :

$$
\frac{\mathrm{d} \sigma_{k q}^{\prime *}}{\mathrm{~d} t}=-\lambda_{k}^{q} \sigma_{k q}^{\prime *}=-\lambda_{k} \sigma_{k q}^{*}
$$

with

$$
\begin{aligned}
\lambda_{k}=\sum_{\substack{l \\
l \leqslant 2 S}} \frac{J_{l}}{\hbar^{2}} & \frac{\left|\left\langle S\left\|R_{l}\right\| S\right\rangle\right|^{2}}{2 S+1} \times \\
& \times(1-(2 S+1) W(S l k S \mid S S)) .
\end{aligned}
$$

Notice that by definition of $T_{1 \mathrm{~s}}, \lambda_{1} \equiv 1 / T_{1 \mathrm{~s}}$ and that if relaxation is due to a single tensorial interaction (given $l$ ), all $\lambda_{k}$ 's are proportional to $1 / T_{1 \mathrm{~s}}$. Let us also recall that the conditions for spherical symmetry of the electronic relaxation have been discussed elsewhere. They are fulfilled by standard conduction electron relaxation in metals and also by certain phonon relaxation processes in high symmetry sites [17].

\section{2 GENERAL EXPRESSIONS OF THE PERTURBATION} FACTOR IN THE PARAMAGNETIC CASE. - In the presence of the degrees of freedom of the electronic spin the general expression for the perturbation factor eq. (15) must be replaced by [18] :

$$
\begin{aligned}
& G_{k^{\prime} k}^{q^{\prime} q}\left(t, t^{\prime}\right)=\frac{1}{(2 S+1)} \times \\
& \quad \times \operatorname{Trace}_{I S}\left\{T_{k}^{q+}(I) \Lambda\left(t, t^{\prime}\right) T_{k^{\prime}}^{q^{\prime}}(I) \Lambda^{+}\left(t, t^{\prime}\right)\right\}
\end{aligned}
$$

where $\Lambda$ now is the evolution operator in the electronuclear manifold $(I, S)$. It is well suited for computa-

$\left.{ }^{2}\right)$ In reality $I$ is also coupled directly to the lattice but this coupling is negligible compared to the electronic spin-lattice coupling. 
tions using a basis of eigenstates of $\mathbf{I}^{2}, \mathbf{S}^{2}, I_{z}, S_{z}$ are eigenstates of $\mathbf{I}^{2}, \mathbf{S}^{2}, \mathbf{F}^{2}=(\mathbf{I}+\mathbf{S})^{2}$ and (decoupled basis $m_{I} m_{S}$ ).

$F_{z}=I_{z}+S_{z}$

On the other hand, in the absence of external magne- (coupled basis $F F_{z}$ ). Tensorial operators adapted to tic field the eigenstates of $A \mathbf{I} . \mathbf{S}$ are $\left|F, F_{z}\right\rangle$ which this coupled basis are [19] :

$$
F^{\prime} F V_{k}^{q}=\sum_{f f^{\prime}}(-1)^{F^{\prime}-f^{\prime}} \sqrt{2 k+1}\left(\begin{array}{ccc}
F^{\prime} & k & F \\
-f^{\prime} & q & f
\end{array}\right)\left|F^{\prime} f^{\prime}\right\rangle\langle F f| ; k \leqslant 2(I+S)
$$

with hermitian conjugate :

$$
\left({ }^{F^{\prime} F} V_{k}^{q}\right)^{+}=(-1)^{F^{\prime}-F-q}\left({ }^{F F^{\prime}} V_{k}^{-q}\right)
$$

and orthogonality properties :

$$
\operatorname{Trace}_{I S}\left\{\left(G^{G^{\prime} G} V_{k^{\prime}}^{q^{\prime}}\right)^{+}\left({ }^{F^{\prime} F} V_{k}^{q}\right)\right\}=\delta_{F^{\prime} G^{\prime}} \delta_{F G} \delta_{k k^{\prime}} \delta_{q q^{\prime}} .
$$

The $T_{k}^{q}(I)$ are related to the ${ }^{F^{\prime} F} V_{k}^{q}$ by (Edmonds [20] eq. (5.4.1) and (7.1.7)) :

with :

$$
T_{k}^{q}(I)=\sum_{F F^{\prime}} F^{\prime} F a_{k}\left(F^{\prime} F V_{k}^{q}\right)
$$

$$
{ }^{F^{\prime} F} a_{k}=(-1)^{I+S+F+k} \sqrt{(2 F+1)\left(2 F^{\prime}+1\right)}\left\{\begin{array}{ccc}
F & F^{\prime} & k \\
I & I & S
\end{array}\right\} .
$$

Inserting these equations into (19) we get a new expression for $G$

$$
G_{k_{1} k_{2}}^{q_{1} q_{2}}(t)=\frac{1}{2 S+1} \operatorname{Trace}\left\{\sum_{\substack{F G \\ F^{\prime} G^{\prime}}}\left({ }^{F^{\prime} G^{\prime}} a_{k_{2}}\right)\left({ }^{F G} a_{k_{1}}\right)\left({ }^{F^{\prime} G^{\prime}} V_{k_{2}}^{q_{2}^{ \pm}}\right) \Lambda\left(t, t^{\prime}\right)\left({ }^{F G} V_{k_{1}}^{q_{1}}\right) \Lambda^{+}\left(t, t^{\prime}\right)\right\}
$$

or alternatively in terms of Heisenberg operators :

$$
G_{k_{1} k_{2}}^{q_{1} q_{2}}(t)=\frac{1}{(2 S+1)} \text { Trace }\left\{\sum_{\substack{F G \\ F^{\prime} G^{\prime}}}\left(F^{F^{\prime} G^{\prime}} a_{k_{2}}\right)\left({ }^{F G} a_{k_{1}}\right)\left({ }^{F^{\prime} G^{\prime}} V_{k_{2}}^{q_{2}}(t)\right)^{+}\left({ }^{F G} V_{k_{1}}^{q_{1}}\right)\right\} .
$$

We now show how $G$ can be deduced from the equation of evolution of the density matrix $\sigma$ of the electronuclear manifold (IS) in the basis $\left|F F_{z}\right\rangle$. Let us perform a multipole expansion of $\sigma$ :

$$
\sigma=\sum_{\substack{F G \\ k q}}\left(\sigma_{k q}^{F G}\right)^{*}\left({ }^{F G} V_{k}^{q}\right)
$$

It is easy to show that :

Consequently :

$$
\begin{aligned}
\sigma_{k_{2} q_{2}}^{F^{\prime} G^{\prime *}}(t) & =\sum_{\substack{F G \\
k_{1} q_{1}}} \operatorname{Trace}\left\{\left({ }^{F^{\prime} G^{\prime}} V_{k_{2}}^{q_{2}}\right)^{+} \Lambda(t)\left({ }^{F G} V_{k_{1}}^{q_{1}}\right) \Lambda^{+}(t)\right\} \sigma_{k_{1} q_{1}}^{F G^{*}}(0) \\
& \equiv \sum_{\substack{F G \\
k_{1} q_{1}}} f_{k_{1} q_{1} \rightarrow k_{2} q_{2}}^{F G \rightarrow F^{\prime} G^{\prime}}(t) \sigma_{k_{1} q_{1}}^{F G^{*}}(0)
\end{aligned}
$$

$$
G_{k_{1} k_{2}}^{q_{1} q_{2}}(t)=\frac{1}{2 S+1} \sum_{\substack{F G \\ F^{\prime} G^{\prime}}}\left(F^{\prime} G^{\prime} a_{k_{2}}\right)\left({ }^{F G} a_{k_{1}}\right) f_{k_{1} q_{1} \rightarrow k_{2} q_{2}}^{F G \rightarrow F^{\prime} G^{\prime}}(t)
$$

where the $f$ are obtained by integrating the equation of motion of $\sigma$.

Notice that $G$ could also be obtained directly by writing the equation of relaxation of $V$ in the Heisenberg representation (while the equation of relaxation of $\sigma$ is written in the Schrödinger representation). In the high temperature case we are considering here, the two relaxation Liouville supermatrices $R$ and $S$ associated to $V$ and $\sigma$ are exactly the same [6] and this second method using $V(t)$ does not require any special care (this would not be true at low temperatures, see [6]). 
2.3 Perturbation FaCtors IN ZeRo EXTERnAl FiELD. - The hamiltonian of the problem is :

$$
\mathscr{H}=\mathscr{H}_{0}+\mathscr{H}_{1}=A \mathbf{I} . \mathbf{S}+\sum_{l p} R_{l}^{p}(S) F^{p} .
$$

The eigenstates of $\mathscr{H}_{0}$ are $\left|F F_{z}\right\rangle$ with eigen-energies :

$$
E_{F}=\frac{A}{2}[F(F+1)-I(I+1)-S(S+1)]
$$

Let us expand $\sigma$ in terms of ${ }^{F G} V_{k}^{q}$. The contribution of $J_{0}$ to $\mathrm{d} \sigma / \mathrm{d} t$ is trivial :

$$
\frac{\mathrm{d}}{\mathrm{d} t} \sigma_{k q}^{F^{\prime} G^{\prime *}}=-\frac{i}{\hbar}\left(E_{F^{\prime}}-E_{G^{\prime}}\right) \sigma_{k q}^{F^{\prime} G^{\prime *}}=-i \omega_{F^{\prime} G^{\prime}} \sigma_{k q}^{F^{\prime} G^{\prime *}}
$$

On the other hand we have shown in the preceding paragraph that, in the absence of nuclear spin, the relaxation of $\mathbf{S}$ is simply described in terms of tensor operators $T_{k}^{q}(S)$. When the nuclear spin is added this will remain true with respect to direct products $T_{k^{\prime}}^{q^{\prime}}(I) T_{k}^{q}(S)$. We shall therefore introduce a multipole expansion adapted to a decoupled basis, work out the relaxation in this basis and go back to the coupled basis.

Let us define :

$$
\left({ }^{k_{I} k_{S}} U_{k}^{q}\right)=\sum_{q_{I} q_{S}}\left\langle k_{I} q_{I} k_{S} q_{S} \mid k q\right\rangle T_{k_{I}}^{q_{I}}(I) T_{k_{S}}^{q_{S}}(S)
$$

The relations between the $V$ and the $U$ are the following [19]

$$
\begin{gathered}
\left({ }^{F G} V_{k}^{q}\right)=\sum_{k_{I} k_{S}} \sqrt{(2 F+1)(2 G+1)\left(2 k_{I}+1\right)\left(2 k_{S}+1\right)}\left\{\begin{array}{lll}
I & I & k_{I} \\
S & S & k_{S} \\
F & G & k
\end{array}\right\}\left({ }^{k_{I} k_{S}} U_{k}^{q}\right) \\
\left({ }^{k_{I} k_{S}} U_{k}^{q}\right)=\sum_{F^{\prime} G^{\prime}} \sqrt{\left(2 F^{\prime}+1\right)\left(2 G^{\prime}+1\right)\left(2 k_{I}+1\right)\left(2 k_{S}+1\right)}\left\{\begin{array}{ccc}
I & I & k_{I} \\
S & S & k_{S} \\
F^{\prime} & G^{\prime} & k
\end{array}\right\}\left({ }^{F^{\prime} G^{\prime}} V_{k}^{q}\right) .
\end{gathered}
$$

When we expand $\sigma$ in terms of ${ }^{k_{I} k_{s}} U_{k}^{q}$ a trivial adaptation of eq. (17) leads to a relaxation term

$$
\frac{\mathrm{d}}{\mathrm{d} t} \sigma_{k q}^{k_{\mathrm{I}} k s^{*}}=-\lambda_{k s} \sigma_{k q}^{k_{1} k^{*}}
$$

Transformation to $\sigma_{k q}^{F G}$ is then achieved by using eq. (35) and (36) and, after defining :

$$
\begin{aligned}
\beta_{k}\left(F G \leftrightarrow F^{\prime} G^{\prime}\right)=\sum_{k_{I} k_{S}} \sqrt{(2 F+1)(2 G+1)\left(2 F^{\prime}+1\right)\left(2 G^{\prime}+1\right)}\left(2 k_{I}+1\right)\left(2 k_{S}+1\right) \times & \times\left\{\begin{array}{ccc}
I & I & k_{I} \\
S & S & k_{S} \\
F & G & k
\end{array}\right\}\left\{\begin{array}{ccc}
I & I & k_{I} \\
S & S & k_{S} \\
F^{\prime} & G^{\prime} & k
\end{array}\right\} \lambda_{k_{S}}
\end{aligned}
$$

we finally get the following equation of motion for $\sigma$ :

$$
\frac{\mathrm{d}}{\mathrm{d} t} \sigma_{k q}^{F^{\prime} G^{\prime *}}=-i \omega_{F^{\prime} G^{\prime}} \sigma_{k q}^{F^{\prime} G^{\prime *}}-\sum_{F G} \beta_{k}\left(F G \leftrightarrow F^{\prime} G^{\prime}\right) \sigma_{k q}^{F G^{*}}
$$

As already mentioned the perturbation factors are obtained by integrating this system, determining the $f_{k_{1} q_{1} \rightarrow k_{2} q_{2}}^{F G \rightarrow F^{\prime} G^{\prime}}$ (eq. (29)) and inserting them into eq. (30).

Notice that when $\lambda_{k_{S}}$ is given by eq. (18) the summation over $k_{I}, k_{S}$ in eq. (38) can be performed with the help of an identity due to Ducloy [38] and eq. (38) becomes :

$$
\begin{aligned}
& \beta_{k}\left(F G \leftrightarrow F^{\prime} G^{\prime}\right)=\sum_{l} \frac{J_{l}}{\hbar^{2}} \frac{\left|\left\langle S\left\|U_{l}\right\| S\right\rangle\right|^{2}}{(2 S+1)} {\left[\delta_{F F^{\prime}} \delta_{G G^{\prime}}+\right.} \\
&+(-1)^{F+F^{\prime}+k+l+1}(2 S+1) \sqrt{(2 F+1)\left(2 F^{\prime}+1\right)(2 G+1)\left(2 G^{\prime}+1\right)} \\
&\left.\times\left\{\begin{array}{ccc}
G^{\prime} & G & l \\
S & S & I
\end{array}\right\}\left\{\begin{array}{lll}
F^{\prime} & F & l \\
S & S & I
\end{array}\right\}\left\{\begin{array}{lll}
F & G & k \\
G^{\prime} & F^{\prime} & l
\end{array}\right\}\right] .
\end{aligned}
$$


Several comments can be made about eq. (39). First, in deriving it we have said that the passage from eq. (17) to eq. (37) was trivial; this is true only as far as the white spectrum approximation is valid, but this condition is precisely realized here since we assume a spherical relaxation of $\mathbf{S}$. Second the damping coefficients $\beta_{k}$ depend on all $\lambda_{k_{s}}$. When $S=1 / 2$ there is only one $\lambda_{k_{s}}: \lambda_{1} \equiv 1 / T_{1 \mathrm{~s}}$. For general $S$ there are $2 S$ of them : $\lambda_{1}, \lambda_{2}, \ldots, \lambda_{2 S}$ which can be considered as independent parameters unless we consider a specific relaxation mechanism (given $l$ ) in which case, by virtue of eq. (18) they can all be expressed as functions of $\lambda_{1}=1 / T_{1 \mathrm{~s}}$. Third in eq. (39) we have coupling only between $\sigma_{k q}^{F G}$,s with the same $k$ and $q$, and for given $k$ the equation is independent of $q$. As will appear later, by comparison with the magnetic field term which is uniaxial, this feature arises from the fact that $\lambda_{k_{s}}^{q_{s}} \equiv \lambda_{k_{\mathrm{s}}}$ is independent of $q_{s}$. A consequence of it is that (see eq. (29) and (30)) :

$$
G_{k k^{\prime}}^{q q^{\prime}}(t)=\delta_{k k^{\prime}} \delta_{q q^{\prime}} G_{k k}^{00}(t) .
$$

Let us consider a specific example : $I=2, S=1 / 2$ which leads to two hyperfine levels $F=5 / 2$ and $F=3 / 2$. Taking account of eq. (40), we only have to compute $G_{22}^{00}(t)$ and $G_{44}^{00}(t)$. On the other hand for given $k$ and $q$ the number of coupled eq. (39) is equal to the number of couples $(F, G)$, i.e. $[\operatorname{Inf}(2 I, 2 S)+1]^{2}$, which here is equal to 4 . In practice we have 4 equations when $k=2$ and only 3 when $k=4$ (since, by virtue of the triangle rule ${ }^{3 / 2,3 / 2} V_{4}^{q}=0$ ). Consequently in order to obtain $G_{22}^{00}$ and $G_{44}^{00}$ we have to solve respectively a system of four linear differential equations and a system of three linear differential equations.

For comparison let us consider what the dimensions of the problem would be if we did not use tensorial operators. In terms of the Liouville evolution superoperator $\overline{\mathcal{V}}(t)$ or reference [6], eq. (19) gives :

$$
G_{k^{\prime} k}^{q^{\prime} q}(t)=\frac{1}{(2 S+1)} \operatorname{Trace}_{I S}\left\{T_{k}^{q^{+}}(I) \overline{\mathcal{V}}(t) T_{k^{\prime}}^{q^{\prime}}(I)\right\}
$$

in which $\bar{V}(t)$ describes the evolution of the density matrix $\sigma$ :

$$
\sigma(t)=\bar{v}(t) \sigma(0) .
$$

In the presence of relaxation $\overline{\mathcal{V}}(t)$ has the general form [6] :

$$
\bar{v}(t)=\exp \left[\left(-\frac{i}{\hbar} \mathscr{H}_{0}^{\times}+R\right) t\right]
$$

where $\mathcal{H}_{0}^{\times}$is the Liouville hamiltonian associated with $\mathcal{H}_{0}$, and $R$ describes the effects of relaxation. The dimensions of the corresponding Liouville matrices are $\left[(2 I+1)^{2}(2 S+1)^{2}\right] \times\left[(2 I+1)^{2}(2 S+1)^{2}\right]$ i.e. $100 \times 100$. In order words $\bar{v}(t)$ would be obtained by solving a system of 100 coupled differential equa- tions. The simplification introduced by the tensor operators is clearly considerable.

However even with this simplification the numerical solution of system (39) and the subsequent computation of the perturbation factor generally require a computer. This will be done in section 3. Before undertaking it we may already check our results in some limiting cases and compare our relaxation model with that of Scherer and Blume.

2.4 COMPARISON WITH OTHER THEORIES. - First in the absence of relaxation, solution of system (39) is trivial and with help of eq. (29) (30) we immediately get the standard expression for the perturbation factor in this case ([7] eq. (96), [18]) :

$$
\begin{aligned}
& G_{k k^{\prime}}^{q q^{\prime}}(t)=\delta_{k k^{\prime}} \delta_{q q^{\prime}} \sum_{F F^{\prime}} \times \\
& \times \frac{(2 F+1)\left(2 F^{\prime}+1\right)}{2 S+1}\left\{\begin{array}{lll}
I & F & S \\
F^{\prime} & I & k
\end{array}\right\}^{2} \mathrm{e}^{-i \omega_{F F^{\prime}} t}
\end{aligned}
$$

Second, in the introduction we have mentioned that the relaxation model of Scherer and Blume for electronic relaxation amounts to taking a transition operator :

$$
\left\langle m_{S}, m_{S}^{\prime}\left|\tau_{\mathrm{av}}\right| m_{S}^{\prime \prime}, m_{S}^{\prime \prime \prime}\right\rangle=\frac{\delta_{m_{S} m_{S}^{\prime}} \delta_{m^{\prime \prime} m^{\prime \prime \prime}}}{2 S+1} .
$$

The corresponding Liouville relaxation operator is [11] :

$$
R=\lambda\left(\tau_{\mathrm{av}}-1\right)
$$

$(\lambda / 2 \pi=$ frequency of the pulses).

If we insert this expression of $R$ into the equation of motion of the density matrix $\sigma^{\prime}$ of the bare electronic spin $(I=0)$ :

$$
\frac{\mathrm{d} \sigma^{\prime}}{\mathrm{d} t}=R \sigma^{\prime}
$$

and if we perform a multipole expansion of $\sigma^{\prime}$ (eq. (16))

$$
\sigma^{\prime}=\sum_{k q} T_{k}^{q}(S) \sigma_{k q}^{\prime *}
$$

we easily arrive at the relaxation equations :

$$
\frac{\mathrm{d} \sigma_{k q}^{\prime *}}{\mathrm{~d} t}=-\lambda \sigma_{k q}^{\prime *}
$$

In other words the Scherer and Blume model amounts to assuming that all multipole components of the electronic spin relax with the same time constant :

$$
\lambda_{k_{s}}^{q_{s}}=\lambda \quad \text { whatever } k_{s}, q_{s} .
$$

One must now ask two questions : first, can this condition be satisfied by microscopic processes in a solid; second, does it correspond to a situation of physical interest? 
We have already seen that for all spherical relaxation processes $\lambda_{k s}^{q s}=\lambda_{k s}$. Then eq. (50) reduces to the system :

$$
\lambda_{k_{s}}=\lambda \text { whatever } k_{s} .
$$

There are $2 S$ values of $k_{s}$ and therefore $2 S$ equations. On the other hand we have shown that (eq. (18)) :

$$
\begin{aligned}
\lambda_{k_{S}}=\sum_{l} \frac{J_{l}}{\hbar^{2}} \frac{\left|\left\langle S\left\|R_{l}\right\| S\right\rangle\right|^{2}}{(2 S+1)} \times \\
\times\left(1-(2 S+1) W\left(S l k_{S} S \mid S S\right)\right)
\end{aligned}
$$

with $l \leqslant 2 S$.

Inserting (52) into (51) we see that we have to solve a system of $2 S$ equations with $2 S$ unknowns which are the quantities $J_{l}$ (or $D_{l}^{-}=\frac{J_{l}}{\hbar^{2}} \frac{\left|\left\langle S\left\|R_{l}\right\| S\right\rangle\right|^{2}}{(2 S+1)}$ ), depending whether we use normalized tensor operators or not). Such a system always has a unique solution. Therefore it is always possible to find one combination of microscopic processes (and one only) for which the theory of Scherer-Blume applies.

On the other hand there is no reason a priori why this precise combination would be of physical interest. There is however an exception which is the simple case $S=1 / 2$. Then in our model there is only one $\lambda_{k_{s}}$ corresponding to $k_{S}=1: \lambda_{1}=1 / T_{1 S}$; in that case if we make the identification $\lambda \leftrightarrow 1 / T_{1 S}$, the perturbation factors computed by our theory or by SchererBlume's theory should be identical. On the contrary if $S=1$ and if we assume for example that the relaxa- tion of $S$ is due to a coupling with the conduction electrons $(l=1)$, we have that $\lambda_{1}=1 / T_{1 \mathrm{~s}}$ and

$$
\lambda_{2}=3 / T_{1 \mathrm{~s}}=3 \lambda_{1} \text {, }
$$

which is not compatible with eq. (50); we therefore expect that the results obtained by the two theories should be different.

Finally, in the limit of very fast relaxation $\lambda_{1} \gg A / \hbar$ we must check that our theory reduces to Abragam and Pound's result [1] :

$$
G_{k}(t)=\mathrm{e}^{-\mu_{k} t}
$$

with

$$
\mu_{k}=\frac{A^{2} k(k+1) S(S+1)}{3} T_{1 \mathrm{~S}} .
$$

The relevant demonstration, as well as a different comparison with Scherer and Blume, are given in the Appendix I.

2.5 Perturbation factor IN THE PResence OF A MAGNETIC FIELD. - In this case we must add to $\mathscr{H}_{0}$ the electronic Zeeman coupling $g \mu_{\mathrm{B}} S_{z} H_{0}$, and in the coupled basis we have to compute commutators of the type :

$$
-\frac{i}{\hbar}\left[\mathcal{H}_{0}, \sigma\right] \rightarrow-\frac{i}{\hbar}\left[S_{z},{ }^{F^{\prime} G^{\prime}} V_{\mathbf{K}}^{Q}\right] .
$$

This is achieved by going from the coupled basis to the decoupled basis and back with the help of eq. (34), (35), (36). We are then led to introduce a coupling coefficient :

$$
\begin{aligned}
& u_{K \boldsymbol{Q} \rightarrow \mathbf{K}^{\prime} \mathbf{Q}}^{F G \rightarrow \boldsymbol{F}^{\prime} \mathbf{G}^{\prime}}=\sum_{\substack{\boldsymbol{k}_{\boldsymbol{I}} \boldsymbol{k}_{\boldsymbol{S}} \\
\boldsymbol{q}_{\mathbf{I}} \boldsymbol{q}_{\boldsymbol{S}}}} \sqrt{(2 F+1)(2 G+1)\left(2 F^{\prime}+1\right)\left(2 G^{\prime}+1\right)}\left(2 k_{I}+1\right)\left(2 k_{S}+1\right) \times \\
& \times\left\{\begin{array}{ccc}
I & I & k_{I} \\
S & S & k_{S} \\
F & G & K
\end{array}\right\}\left\{\begin{array}{ccc}
I & I & k_{I} \\
S & S & k_{S} \\
F^{\prime} & G^{\prime} & K^{\prime}
\end{array}\right\} \\
& \times\left\langle k_{I} q_{I} k_{S} q_{S} \mid K Q\right\rangle\left\langle k_{I} q_{I} k_{S} q_{S} \mid K^{\prime} Q\right\rangle q_{S} \text {. }
\end{aligned}
$$

In terms of $u$, the equation of motion of $\sigma$ is :

$$
\frac{\mathrm{d}}{\mathrm{d} t}\left({ }^{F G} \sigma_{k}^{q^{*}}\right)=-\frac{i}{\hbar}\left(E_{F}-E_{G}\right)\left({ }^{F G} \sigma_{k}^{q^{*}}\right)-\frac{i}{\hbar} g \mu_{\mathrm{B}} H_{0} \sum_{\boldsymbol{k}^{\prime} F^{\prime} G^{\prime}} u_{k q \leftrightarrow k^{\prime} q}^{F G \leftrightarrow G^{\prime} G^{\prime}}\left({ }^{\prime} G^{\prime} \sigma_{k^{\prime}}^{q^{*}}\right)-\sum_{F^{\prime} G^{\prime}} \beta_{k}^{F G \leftrightarrow F^{\prime} G^{\prime}}\left({ }^{F^{\prime} G^{\prime}} \sigma_{k}^{q^{*}}\right) .
$$

It appears that now the only good quantum number of the problem is $q$, in other words we have couplings between $\sigma_{k q}^{F G}$ with different $k$; moreover the coefficient $u_{k q \leftrightarrow k^{\prime} q}^{F G \leftrightarrow G^{\prime} G^{\prime}}$ depends on $q$. The perturbation factors of interest in PAC will be all the $G_{k k^{\prime}}^{q q}$ with $k, k^{\prime}$ even.

As an example let us assume that $I=2, S=1 / 2$. In a perpendicular geometry $\left(\mathbf{k}_{1}, \mathbf{k}_{2} \perp \mathbf{H}_{0}\right)$ we must compute $G_{22}^{00}, G_{22}^{22}, G_{24}^{00}, G_{24}^{22}, G_{24}^{24}, G_{44}^{00}, G_{44}^{22}, G_{44}^{24}$, $G_{44}^{44}$ and similar terms obtained by reversing the order of $k$ or the sign of $q$. The dimensions of the differential systems associated with the different $q$ 's are : 18 for $q=0(16$ for $q= \pm 1), 12$ for $q= \pm 2,(8$ for $q= \pm 3)$, 4 for $q= \pm 4$ ( 1 for $q= \pm 5$ ).

It appears that the use of the coupled basis and of tensor operators has little interest in this case, this being clearly due to the fact that the external field lowers the symmetry from spherical to uniaxial. In addition this basis is not adapted to the high field 
case $\left(\mu_{\mathrm{B}} H_{0} \gg A\right)$ where we may expect to recover, in the absence of relaxation the well known formula [21] :

$$
\begin{aligned}
G_{k k^{\prime}}^{q q^{\prime}}(t)= & \frac{1}{2 S+1} \delta_{k k^{\prime}} \delta_{q q^{\prime}} \frac{\sin \left[q \frac{A}{\hbar}\left(S+\frac{1}{2}\right) t\right]}{\sin \left[q \frac{A}{\hbar} \frac{1}{2} t\right]} \equiv \\
& \equiv \frac{1}{2 S+1} \sum_{m_{S}=-S}^{+S} \exp \left(-i q \frac{A}{\hbar} m_{S} t\right)
\end{aligned}
$$

and in the presence of relaxation the results of diagonal stochastic theories of the Anderson Weiss type [3]. Let us check these two points.

2.6 LiMITING VALUES OF THE PERTURBATION FACTORS IN HIGH FIELD. - In this paragraph, as well as in section 4, we shall work in the decoupled basis $m_{I} m_{S}$ which is more convenient for high field calculations. Furthermore, in view of the physical discussion we will compute the Laplace-Fourier transforms $\widetilde{G}_{k k^{\prime}}^{q q^{\prime}}(\omega)$ of the perturbation factors (= Laplace transforms in which we take $p=-i \omega)$. According to eq. (41) and (43) :

$$
G_{k^{\prime} k}^{q^{\prime} q}(t)=\frac{1}{(2 S+1)} \sum_{\substack{m_{I}, m_{I}^{\prime} \\ n_{I}, n_{I}^{\prime} \\ m_{S}, n_{S}}}\left\{\left\langle m_{I}^{\prime}\left|T_{k}^{q+}(I)\right| m_{I}\right\rangle\left\langle n_{I}\left|T_{k^{\prime}}^{q^{\prime}}(I)\right| n_{I}^{\prime}\right\rangle \times\left\langle m_{I} m_{S}, m_{I}^{\prime} m_{S}|\overline{\mathcal{V}}(t)| n_{I} n_{S}, n_{I}^{\prime} n_{S}\right\rangle\right\}
$$

and

$$
\begin{aligned}
\tilde{G}_{k^{\prime} k}^{q^{\prime} q}(\omega)=\frac{1}{(2 S+1)} \sum_{\substack{m_{I}, m_{I}^{\prime} \\
n_{I} n_{I}^{\prime} \\
m_{S}, n_{S}}}\left\{\left\langle m_{I}^{\prime}\left|T_{k}^{q+}\right| m_{I}\right\rangle\left\langle n_{I}\left|T_{k^{\prime}}^{q^{\prime}}(I)\right| n_{I}^{\prime}\right\rangle \times\right. & \left.\times\left\langle m_{I} m_{S}, m_{I}^{\prime} m_{S}\left|\frac{-1}{i \omega-\frac{i}{\hbar} \mathcal{H}_{0}^{\times}+R}\right| n_{I} n_{S}, n_{I}^{\prime} n_{S}\right\rangle\right\} .
\end{aligned}
$$

Let us first consider the truncated hamiltonian :

$$
\mathcal{H}_{0}^{\prime}=A I_{z} S_{z}+g \mu_{\mathrm{B}} H_{0} S_{z}
$$

In the decoupled basis it is diagonal. Consequently in the Liouville representation we have :

$$
\begin{aligned}
\left\langle m_{I} m_{S}, m_{I}^{\prime} m_{S}^{\prime}\left|\left(A I_{z} S_{z}+g \mu_{\mathrm{B}} H_{0} S_{z}\right)^{\times}\right| n_{I} n_{S}, n_{I}^{\prime} n_{S}^{\prime}\right\rangle= & \delta_{m_{I}, n_{I}} \delta_{m_{I}^{\prime}, n_{I}^{\prime}} \delta_{m_{S}, n_{S}} \delta_{m_{S}^{\prime}, n_{S}^{\prime}} \times \\
& {\left[g \mu_{\mathrm{B}} H_{0}\left(m_{S}-m_{S}^{\prime}\right)+A\left(m_{I} m_{S}-m_{I}^{\prime} m_{S}^{\prime}\right)\right] . }
\end{aligned}
$$

On the other hand $R$ describes the relaxation of the electronic spin $\mathbf{S}$

$$
\left\langle m_{I} m_{S}, m_{I}^{\prime} m_{S}^{\prime}|R| n_{I} n_{S}, n_{I}^{\prime} n_{S}^{\prime}\right\rangle=\delta_{m_{I}, n_{I}} \delta_{m_{I}^{\prime}, n_{I}^{\prime}}\left\langle m_{S} m_{S}^{\prime}|R| n_{S}, n_{S}^{\prime}\right\rangle .
$$

It follows from these two equations that if we neglect the off diagonal part of the hyperfine interaction $\left(\mathcal{H}_{0} \rightarrow \mathcal{H}_{0}^{\prime}\right)$ the Liouville matrix of $i \omega-\frac{i}{\hbar} \mathcal{H}_{0}^{\prime \times}+R$ factorizes into submatrices with dimensions

$$
(2 S+1)^{2} \times(2 S+1)^{2}
$$

corresponding to given values of $m_{I}$ and $m_{I}^{\prime}$ in eq. (60) and (61).

In particular when $S=1 / 2$ and when its relaxation is spherical, it is entirely described by one relaxation parameter corresponding to $k=1: \lambda_{1} \equiv 1 / T_{1 \mathrm{~s}}$. In that case the electronic relaxation matrix $R$ has the form :

$$
\left[\begin{array}{cccc}
-1 / 2 T_{1 \mathrm{~s}} & +1 / 2 T_{1 \mathrm{~s}} & 0 & 0 \\
+1 / 2 T_{1 \mathrm{~s}} & -1 / 2 T_{1 \mathrm{~s}} & 0 & 0 \\
0 & 0 & -1 / T_{1 \mathrm{~s}} & 0 \\
0 & 0 & 0 & -1 / T_{1 \mathrm{~s}}
\end{array}\right]
$$

and the submatrices of $i \omega-\frac{i}{\hbar} \mathcal{H}_{0}^{\prime x}+R$ for given $m_{I}$ and $m_{I}^{\prime}$ are $4 \times 4$ and are given on top of next page. When we take the inverse of this matrix and insert it into eq. (59) the presence of the factor $\left\langle m_{I}^{\prime}\left|T_{k}^{q+}\right| m_{I}\right\rangle$ introduces the condition

$$
m_{I}-m_{I}^{\prime}=q .
$$

In addition it follows from the selection rules on $m_{S}$ and $n_{S}$ in eq. (59) that we only need the inverse of the $2 \times 2$ matrix in the upper left quarter (which we shall call matrix of type I) :

$$
(I)=\left[\begin{array}{c:c}
i \omega-\frac{i q A}{2 \hbar}-\frac{1}{2 T_{1}} & \frac{1}{2 T_{1 \mathrm{~s}}} \\
\hdashline \frac{1}{2 T_{1 \mathrm{~s}}} & i \omega+\frac{i q A}{2 \hbar}-\frac{1}{2 T_{1 \mathrm{~s}}}
\end{array}\right]
$$




\begin{tabular}{|c|c|c|c|c|}
\hline & ++ & -- & +- & -+ \\
\hline++ & $\begin{array}{c}i \omega-\frac{i}{2} \frac{A}{\hbar}\left(m_{I}-m_{I}^{\prime}\right) \\
-\frac{1}{2 T_{1 \mathrm{~s}}}\end{array}$ & $\frac{1}{2 T_{1 \mathrm{~s}}}$ & & \\
\hline-- & $\frac{1}{2 T_{1 \mathrm{~S}}}$ & $\begin{array}{c}i \omega+\frac{i}{2} \frac{A}{\hbar}\left(m_{I}-m_{I}^{\prime}\right) \\
-\frac{1}{2 T_{1 \mathrm{~S}}}\end{array}$ & & \\
\hline+- & & & $\begin{array}{l}-i g \mu_{\mathrm{B}} H_{0} / \hbar \\
-i \frac{A}{2 \hbar}\left(m_{I}+m_{I}^{\prime}\right) \\
-\frac{1}{T_{1 \mathrm{~S}}}\end{array}$ & \\
\hline-+ & & & & $\begin{array}{c}+i g \mu_{\mathrm{B}} H_{0} / \hbar \\
+\frac{i A}{2 \hbar}\left(m_{I}+m_{I}^{\prime}\right) \\
-\frac{1}{T_{1 \mathrm{~S}}}\end{array}$ \\
\hline
\end{tabular}

while the one-by-one submatrices of the lower right quarter (matrices of type II) do not contribute to the perturbation factor.

It is quite apparent from inspection of matrix (I) that in the absence of relaxation $\left(1 / T_{1 \mathrm{~S}}=0\right)$ we will recover eq. (57) with $S=1 / 2$ and that in the presence of relaxation, matrix (I) being identical to the standard matrix $A$ of simple stochastic theories ([8] Chap. 10, p. 447) our formulas will also be in agreement with previous work in this field [3]. Similar results would be obtained for $S>1 / 2$.

On the contrary when we add the off-diagonal terms of the hyperfine interaction, they will connect a matrix of type I with indices $m_{I}, n_{I}$ to matrices of type II with indices $m_{I} \pm 1, n_{I} \pm, \mp 1$. The Liouville matrix

$$
i \omega-\frac{i}{\hbar} \mathfrak{H}_{0}^{\times}+R
$$

does not factorize any longer and things become more complicated. However, since matrices of type II contain $g \mu_{\mathrm{B}} H_{0} \gg A$, the corrections introduced by the off-diagonal terms of $A \mathbf{I} . \mathbf{S}$ will be of order $\frac{A^{2}}{g \mu_{\mathrm{B}} H_{0}}$ i.e. small. This point will be discussed in more detail in section 4 .

3. PAC experiments in zero magnetic field. Numerical results and discussion. - In what follows we shall first show the numerical results which we have obtained for the perturbation factors $G_{k k}^{q q}(t)$ and compare them with the values derived from the theory of Blume and Scherer. We shall then discuss the possibility of expe- rimental verification of our predictions; it will appear that detailed checking by DPAC experiments is not presently possible because the time resolution of the electronics is insufficient. On the contrary IPAC experiments should be easy to perform since the correlation does not rotate and we indicate the results to be expected in two specific cases.

3.1 DPAC. - In this case the perturbation factors are obtained by first solving the system (39) to obtain the quantities $f$ in eq. (29) which are subsequently inserted into eq. (30). Most calculations have been performed for a nuclear spin $I=2$ which is the lowest value for which both $G_{22}$ and $G_{44}$ are non-zero.

3.1.1 Case $S=1 / 2, I=2$. - When $S=1 / 2$, we have shown that our theory is equivalent to the Scherer-Blume theory with the identification

$$
\lambda_{1} \equiv 1 / T_{1 \mathrm{~s}}=\lambda \text {. }
$$

We have therefore evaluated $G$, both with our own equations and by inverse Laplace transformation of the eq. (8) of Blume, and checked that the results were identical. The results for $G_{22}(t)$ and $G_{44}(t)$ are reported in figure (1.2) and exhibit the expected evolution from a purely oscillating behaviour to a purely damped behaviour (the unit of time is the hyperfine period $T=2 h / 5 A)$. Notice that in this case we are able to compute $G_{k k}(t)$ for arbitrary relaxation time with no more parameters than in the AbragamPound theory; but the Laplace transform of $G_{k k}(t)$ no longer has the simple Abragam Pound form (eq.. (A.19) of the appendix I)

$$
G_{k k}(p)_{\mathrm{AP}}=1 /\left(p+\mu_{k}\right)
$$




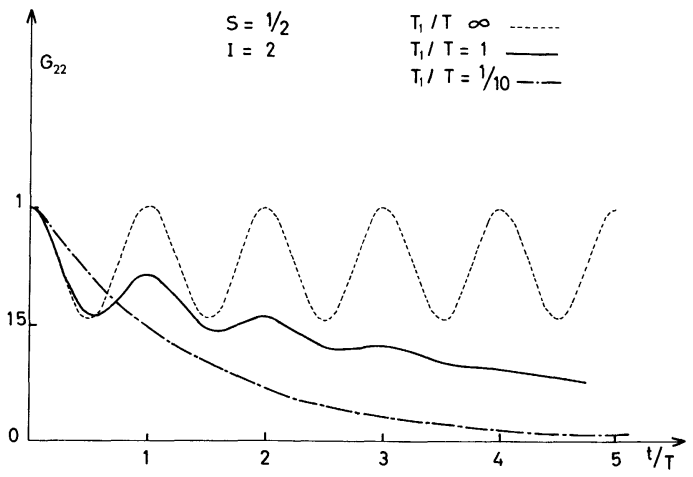

FIG. 1 . $-I=2, S=1 / 2$. Time variation of $G_{22}(t)$ for various values of the electronic relaxation time $T_{1 \mathrm{~s}}$. Time unit is the hyperfine period $T=2 \mathrm{~h} / 5 \mathrm{~A}$.

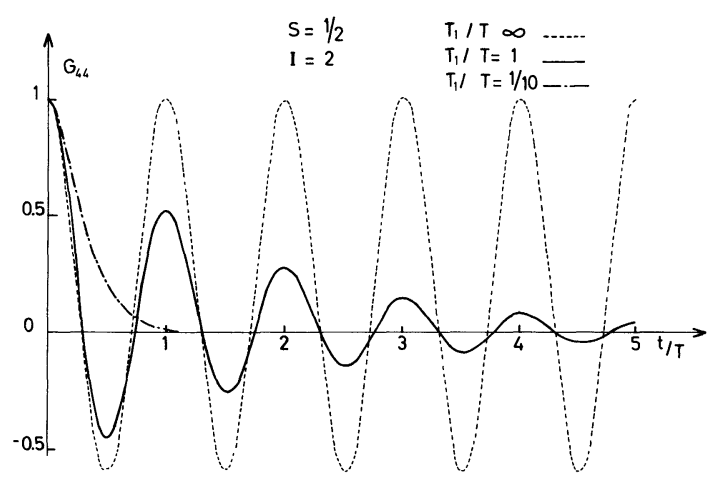

FIG. 2. $-I=2, S=1 / 2$. Time variation of $G_{44}(t)$ for various values of $T_{1 \mathrm{~s}}$.

it is now given by Blume's relation (eq. (6) of the present paper), i.e. in practice it is the ratio of a second order polynomial in $p$ and a third order polynomial in $p$.

3.1.2 Case $S=1, I=2$. - With these values we have three hyperfine levels $F=3,2,1$ and three hyperfine intervals

$$
\hbar \omega_{32}=3 \mathrm{~A}, \quad \hbar \omega_{21}=2 \mathrm{~A}, \quad \hbar \omega_{31}=5 \mathrm{~A} .
$$

On the other hand there are two electronic relaxation parameters $\lambda_{1} \equiv 1 / T_{1 \mathrm{~s}}$ and $\lambda_{2}$.

We have computed $G_{22}$ and $G_{44}$ under the assumption that relaxation was due to a tensorial relaxation of order one (relaxation by a fluctuating field, for example by the conduction electrons); then

$$
\lambda_{1}=1 / T_{1 \mathrm{~s}} \text { and } \lambda_{2}=3 / T_{1 \mathrm{~s}} \text {. }
$$

The results are reported in figures 3 and 4 (time unit $T=h(5 A)$. These figures also contain the curves relative to the Scherer Blume theory with $\lambda=1 / T_{1 \mathrm{~s}}$. As expected the curves relative to the intermediate range $T_{1 \mathrm{~s}}=T$ do not coincide (however, as shown in the appendix $\mathrm{I}$, they do coincide in the Abragam Pound limit).

It is possible to show that in order to reproduce the Scherer Blume curve one must take an adequate

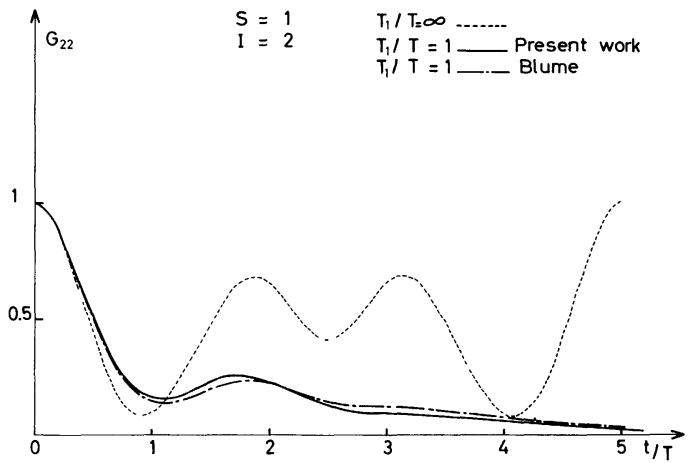

FIG. 3. $-I=2, S=1$. Time variation of $G_{22}(t)$ assuming either relaxation by a fluctuating field (full curve) or Scherer-Blume model with $\lambda \equiv 1 / T_{1 \mathrm{~s}}(-$. - . curve).

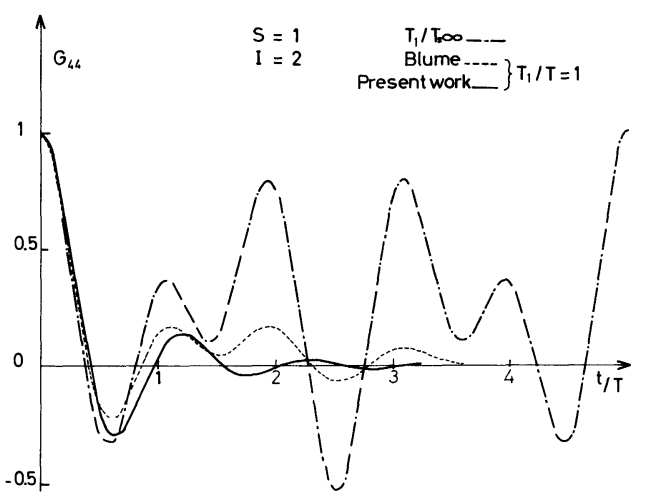

FIG. 4. $-I=2, S=1$. Time variation of $G_{44}(t)$ assuming either relaxation by a fluctuating field (full curve) or Scherer-Blume model with $\lambda \equiv 1 / T_{1 \mathrm{~S}}$ (dashed curve).

mixing of interactions of order $l=1$ and $l=2$. Indeed, in terms of the quantities

$$
D_{l}=\frac{J_{l}}{\hbar^{2}} \frac{\left|\left\langle S\left\|R_{l}\right\| S\right\rangle\right|^{2}}{(2 S+1)}
$$

we must have :

$$
\begin{aligned}
\lambda_{1}=\lambda= & D_{1}[1-(2 S+1) W(S 11 S \mid S S)]+ \\
& +D_{2}[1-(2 S+1) W(S 21 S \mid S S)] .
\end{aligned}
$$

and

$$
\begin{aligned}
\lambda_{2}=\lambda= & D_{1}[1-(2 S+1) W(S 12 S \mid S S)]+ \\
& +D_{2}[1-(2 S+1) W(S 22 S \mid S S)]
\end{aligned}
$$

whose solution is

$$
D_{1}=\frac{1}{3} \lambda \quad \text { and } \quad D_{2}=\frac{5}{9} \lambda \quad \text { or } \quad \frac{D_{2}}{D_{1}}=\frac{5}{3} .
$$

3.1.3 Possibilities of experimental checking. It requires that one be able to observe oscillations at the hyperfine frequencies, i.e. at least four points in the hyperfine period. At the present time the best resolution achieved in DPAC experiments is

$$
\frac{1}{3} \mathrm{~ns}=3 \times 10^{-10} \mathrm{~s} \text {. }
$$


Experiment is therefore limited to hyperfine frequencies less than $10^{3} \mathrm{MHz}$, i.e. essentially to the iron group and the $S$ state ions of the rare earth group. In addition the nuclear lifetime should be long compared to the hyperfine period : $\tau_{\mathrm{n}}>10^{-9} \mathrm{~s}$. Possible candidates satisfying these conditions would be ${ }_{26} \mathrm{Fe}^{57}$ (parent ${ }_{27} \mathrm{Co}^{57}$, cascade $122 \rightarrow 14 \mathrm{keV}$, intermediate state : $I=3 / 2^{-}, E=14.4 \mathrm{keV}$, $\tau_{\mathrm{n}}=1.45 \times 10^{-7} \mathrm{~s}$ ) and ${ }_{64} \mathrm{Gd}^{155}$ (parent ${ }_{63} \mathrm{Eu}^{156}$ or ${ }_{65} \mathrm{~Tb}^{156}$, cascade $180 \mathrm{keV} \rightarrow 87 \mathrm{keV}$, intermediate state : $\left.I=3 / 2^{+}, E=87 \mathrm{keV}, \tau_{\mathrm{n}}=9.08 \times 10^{-9} \mathrm{~s}\right)$.

In order to observe the slow relaxation limit one could try to operate on trivalent iron in selected insulating matrices $\left(^{3}\right)$ where this limit has already been attained in Mössbauer experiments (in a metal its relaxation would be too fast). For Gd, which is always trivalent, slow relaxation around $4 \mathrm{~K}$ should be possible even in a metal. There is however a major difficulty because, in addition to their high electronic spins $\left(S=5 / 2\right.$ for $\mathrm{Fe}^{3+}$ and $S=7 / 2$ for $\mathrm{Gd}^{3+}$ ), these two $S$ state ions usually have a spin hamiltonian which is comparable to their hyperfine structure (numerical values can be found in Abragam and Bleaney [34]) (p. 440 for $\mathrm{Fe}^{3+}$ and p. 335 for $\left.\mathrm{Gd}^{3+}\right)$. The formulas derived in the present paper are not adapted to this situation; on the other hand, in the presence of the spin hamiltonian the dimensions of the differential system or Liouville matrix relevant to the problem might be rather large.

3.2 IPAC. - In this technique one observes the quantities $\left(\tau_{\mathrm{n}}=\right.$ nuclear lifetime of state $\left.I\right)$ :

$$
\frac{1}{\tau_{\mathrm{n}}} \tilde{G}_{k k^{\prime}}^{q q^{\prime}}\left(\frac{1}{\tau_{\mathrm{n}}}\right)=\frac{1}{\tau_{\mathrm{n}}} \int_{0}^{\infty} \mathrm{e}^{-t / \tau_{\mathrm{n}}} G_{k k^{\prime}}^{q q^{\prime}}(t) \mathrm{d} t .
$$

The information obtained in this way is much more limited than in DPAC, but there are almost no experimental limitations. In particular since the correlation does not rotate in zero field, the parameter $\frac{A}{\hbar} \tau_{\mathrm{n}}$ may have an arbitrary value.

Theoretical values of $\tilde{G}_{k k^{\prime}}^{q q^{\prime}}\left(1 / \tau_{n}\right)$ can be obtained :

- when $S=1 / 2$ by use of the Scherer-Blume relationship :

$$
\begin{aligned}
\tilde{G}_{k k^{\prime}}^{q q^{\prime}}\left(\frac{1}{\tau_{\mathrm{n}}}\right)= & \delta_{k k^{\prime}} \delta_{q q^{\prime}} \tilde{G}_{k}\left(\frac{1}{\tau_{\mathrm{n}}}\right)= \\
= & \frac{\tilde{G}_{k}^{0}\left(\frac{1}{\tau_{\mathrm{n}}}+\frac{1}{T_{1 \mathrm{~s}}}\right)}{1-\frac{1}{T_{1 \mathrm{~S}}} \tilde{G}_{k}^{0}\left(\frac{1}{\tau_{\mathrm{n}}}+\frac{1}{T_{1 \mathrm{~s}}}\right)} \delta_{k k^{\prime}} \delta_{q q^{\prime}}
\end{aligned}
$$

- when $S \neq 1 / 2$ by Laplace transformation of the differential system (39).

$\left({ }^{3}\right)$ Notice that in insulators, the radioactive decay may give rise to troublesome after effects.
3.2.1 Case $S=1 / 2$. - The hyperfine interval is $\hbar \omega=\frac{A(2 I+1)}{2}$. We have assumed $I=2$ and computed the values of $\left(1 / \tau_{n}\right) \tilde{G}_{2}\left(1 / \tau_{n}\right)$ as a function of $1 / \omega T_{1 \mathrm{~s}}$ for several values of the parameter $\omega \tau_{\mathrm{n}}$

$$
\omega \tau_{\mathrm{n}}=0.1 ; 1 ; 2 ; 5 ; 10 ; 100 \text {. }
$$

The results are plotted in figure 5. They show that when $1 / \omega T_{1 \mathrm{~s}} \gg 1$ one recovers the results of the Abragam-Pound theory. On the other hand when $\omega \tau_{\mathrm{n}} \ll 0.1$ and $T_{1 \mathrm{~s}} \gg \tau_{\mathrm{n}}$ one should observe the Gabriel effect [22], [14], that is, the perturbation factor is given by the Abragam and Pound formula in which $T_{1 \mathrm{~s}}$ is replaced by $\tau_{\mathrm{n}}$ :

$$
\tilde{G}_{k}\left(\frac{1}{\tau_{\mathrm{n}}}\right)=\frac{1}{\frac{1}{\tau_{\mathrm{n}}}+\frac{A^{2}}{\hbar} \frac{S(S+1)}{3} k(k+1) \tau_{\mathrm{n}}} .
$$

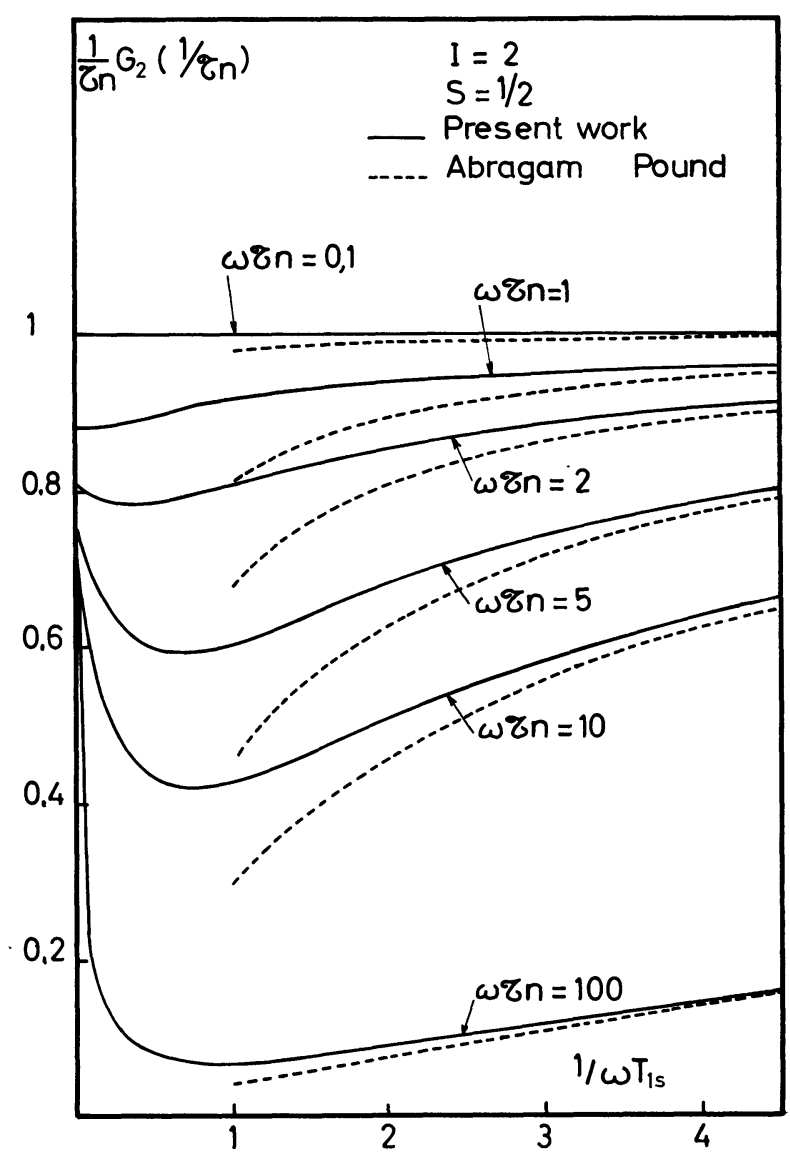

Fig. 5. $-I=2, S=1 / 2, \omega=(A / \hbar)(I+1 / 2)$. Integral perturbation factor : comparison between the present work and the Abragam Pound theory for various values of $\omega \tau_{\mathrm{n}}$.

For $\omega \tau_{\mathrm{n}}=0.1, \tilde{G}_{k}\left(1 / \tau_{\mathrm{n}}\right)$ should therefore become independent of $1 / \omega T_{1 \mathrm{~s}}$ as soon as $1 / \omega T_{1 \mathrm{~s}}<5$. This is indeed that happens.

On an experimental point of view the situation $I=2, S=1 / 2$ is realized for several rare earth ions when embedded in a gold matrix ( $S$ being the effective spin of their lowest electronic state) : $87 \mathrm{keV}$ state 
of $\left({ }_{66} \mathrm{Dy}^{160}\right)^{3+}, 81 \mathrm{keV}$ state of $\left({ }_{68} \mathrm{Er}^{166}\right)^{3+}$ (but the first excited electronic state of $\mathrm{Er}^{3+}$ is at $18 \mathrm{~K}$ only).

Other interesting cases with $S=1 / 2$ are $\mathrm{Yb}^{3+}$ and $\mathrm{Ce}^{3+}$ in gold. ${ }_{70} \mathrm{Yb}^{172}$ has two nuclear states which can be studied by PAC. The first state has $I=3^{+}, \mu=0.64 \mu_{\mathrm{N}}, E=1174 \mathrm{keV}, \tau_{\mathrm{n}}=11.5 \times 10^{-9} \mathrm{~s}$ and the corresponding $\gamma-\gamma$ correlation is $91 \mathrm{keV}$ $1095 \mathrm{keV}$. The second state has $I=2^{+}, \mu=0.6 \mu_{\mathrm{N}}$, $E=79 \mathrm{keV}, \tau_{\mathrm{n}}=2.37 \times 10^{-9} \mathrm{~s}$ and the corresponding correlation is $1095 \mathrm{keV}-79 \mathrm{keV} .{ }_{70} \mathrm{Yb}^{172}$ is created by electronic capture by ${ }_{71} \mathrm{Lu}^{172}$ or by $\beta^{-}$ decay from ${ }_{69} \mathrm{Tm}^{172}$. The lowest electronic state of $\mathrm{Yb}^{3+}$ in gold is $\Gamma_{7}$, as shown by EPR and Mössbauer measurements [5]. Taking account of these previous results and of the nuclear moments of the states $I=3^{+}$and $I=2^{+}$we find that the parameters $\omega \tau_{\mathrm{n}}$ for these two states are respectively $\omega \tau_{\mathrm{n}}=144$ and $\omega \tau_{\mathrm{n}}=30$. The electronic relaxation time $T_{1 \mathrm{~s}}$ inside $\Gamma_{7}$ has been determined by Imbert and Gonzalez on the Mössbauer isotope $\mathrm{Yb}^{170}$, from $1 \mathrm{~K}$ to about $30 \mathrm{~K}$ [5], [15], [16]. As a first approximation [5] $1 / T_{1 \mathrm{~s}}$ is a linear function of temperature (electronic Korringa relaxation) :

$$
\frac{1}{T_{1 \mathrm{~S}}}=\frac{4 \pi}{\hbar}\left(J_{s f} n\left(E_{\mathrm{F}}\right)\right)^{2}\left(\frac{g_{J-1}}{g_{J}}\right)^{2} g^{2} k_{\mathrm{B}} T
$$

and using the experimental values of [5] one should have for both nuclear states of $\mathrm{Yb}^{172}$ :

$$
\frac{1}{\omega T_{1 \mathrm{~s}}}=0.087 \mathrm{~T}
$$

(since the values of $\omega$ for both states are nearly equal).

Consequently we can compute the integrated perturbation factor of $\mathrm{Yb}^{172}$ for both states as a function of temperature without any adjustable parameter. The curves relative to $\left(1 / \tau_{n}\right) G_{22}\left(1 / \tau_{n}\right)$ are represented in figures (6), (7). They exhibit an important deviation with respect to an Abragam-Pound law, which should be observable at low temperatures if the coefficient $A_{2}$ in the unperturbed anisotropy :

$$
W(\theta)=1+A_{2} P_{2}(\cos \theta)+A_{4} P_{4}(\cos \theta)+\cdots
$$

is large enough. This seems indeed to be the case, $A_{2}$ being about $30 \%$ in both cases [23-24], [23-27]; one should however mention that the published experimental results exhibit rather large discrepancies (especially for the cascade $1095-79 \mathrm{keV}$ [23-27]).

It is also worthwhile mentioning that electronic capture or $\beta^{-}$decay might produce an initial population of the excited electronic states of $\mathrm{Yb}^{3+}$. In metals these excited states should generally decay to the ground electronic state in a time much shorter than $T_{1 \mathrm{~s}}$ [29]. Nevertheless, if one wants to avoid these rearrangement effects, it is better to choose a nuclear state which is fed through an upper state with a known relatively long lifetime. This is the case

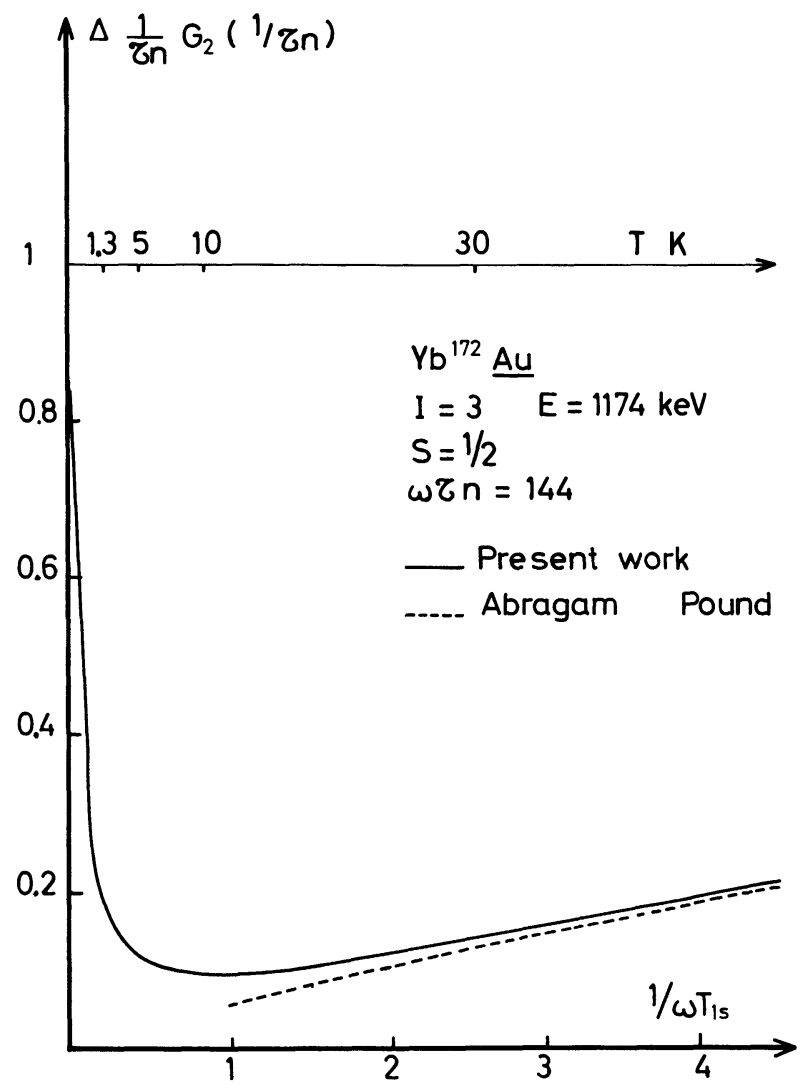

FIG. 6. - Theoretical integral perturbation factor for the $1174 \mathrm{keV}$ state of $\mathrm{Yb}^{172} \mathrm{Au}\left(I=3, S=1 / 2, \omega=(A / \hbar)(I+1 / 2), \omega \tau_{\mathrm{n}}=144\right)$. The temperature scale is based on the values of $1 / T_{1 \mathrm{~s}}$ obtained by Imbert and Gonzalez (neglecting the deviation with respect to linearity in $T$ ).

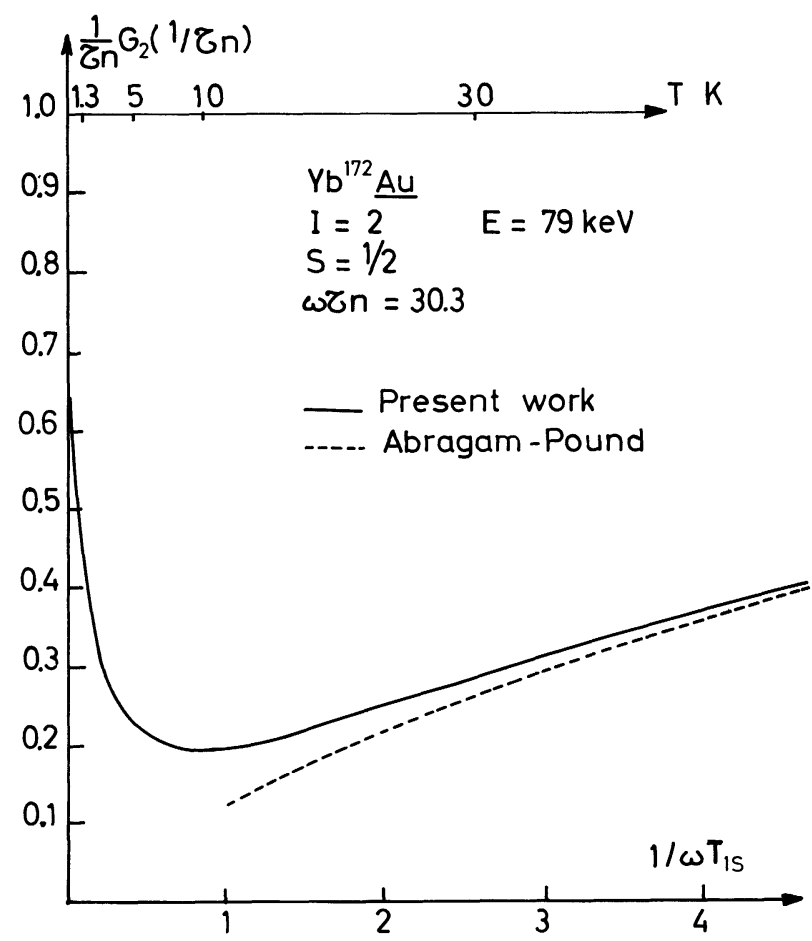

FIG. 7. - Theoretical integral perturbation factor for the $79 \mathrm{keV}$ state of $\mathrm{Yb}^{172} \mathrm{Au}\left(I=2, S=1 / 2, \omega=(A / \hbar)(I+1 / 2), \omega \tau_{\mathrm{n}}=30.3\right)$. 
for the state $I=2^{+}, E=79 \mathrm{keV}$ which is fed by the state $I=3^{+}, E=1174 \mathrm{keV}$ for which $\tau_{\mathrm{n}}=11.5 \mathrm{~ns}$. On the contrary, the lifetime of the states above this last level are not known. If they happen to be of the order of $10^{-11} \mathrm{~s}$ or less, rearrangement problems might be troublesome.

${ }_{58} \mathrm{Ce}^{140}$ has a state $I=4^{+}, \mu=4.06 \mu_{\mathrm{N}}$, $E=2083 \mathrm{keV}, \tau_{\mathrm{n}}=4.92 \times 10^{-9} \mathrm{~s}$ and the corresponding $\gamma-\gamma$ correlation is $328-487 \mathrm{keV} .{ }_{58} \mathrm{Ce}^{140}$ is created by $\beta^{-}$decay from ${ }_{57} \mathrm{La}^{140}$ ( 40.2 hours); the $2412 \mathrm{keV}$ state which feeds the $4^{+}, 2083 \mathrm{keV}$ state has a lifetime of about $10^{-10} \mathrm{~s}$. Nuclear orientation studies [29] have shown that the lowest electronic state of $\mathrm{Ce}^{3+}$ in gold is $\Gamma_{7}$ resulting in a hyperfine parameter $\omega \tau_{\mathrm{n}}=133$. The electronic relaxation time inside $\Gamma_{7}$ is not known. However, if we assume $\left({ }^{4}\right)$ that the (conduction electron-localized spin) coupling $J_{s f}$ has approximately the same value for $\mathrm{Ce}^{3+}$ and $\mathrm{Yb}^{3+}$ we may use eq. (70) to obtain a rough estimation of it. Taking account of the fact that for $\mathrm{Yb}^{3+}$ : $g=24 / 7, g_{J}=8 / 7$ and for $\mathrm{Ce}^{3+}: g=10 / 7$ and $g_{J}=6 / 7$, we arrive at :

$$
\frac{1}{\omega T_{1 \mathrm{~s}}} \sim 0.030 T
$$

The curve of (8) has been computed with this figure. It appears that by going down to $1.3 \mathrm{~K}$ one could study in detail the part of the curve with upward curvature, at least if the coefficient $A_{2}$ in eq. (72) is large enough. Let us check this point.

If the $328-487 \mathrm{keV}$ cascade is $M_{1}, E_{2}(3(1) 4(2), 2)$, the calculated values of $A_{2}$ and $A_{4}$ are :

$$
A_{2}=-0.14, A_{4}=0 \text {. }
$$

The numerous experimental results on this cascade [30-32] lead to an average value of $A_{2} \simeq-0.1$ while $A_{4} \simeq 0$. The difference between the calculated and experimental results might be due to some extranuclear perturbation on the nucleus or to a slight admixture $E_{2} / M_{1}$ of the first transition [32]. If we assume the value of $A_{2}$ to be -0.1 , the maximum variation of $W(\theta)$ is $(3 / 2) A_{2}=-0.15$. Accordingly the expected maximum variation of $W(\theta)$ for ${ }_{58} \mathrm{Ce}^{140} \mathrm{Au}$, as predicted by our theory is -0.10 at $1.3 \mathrm{~K}$ and -0.02 at $30 \mathrm{~K}$. This thermal variation of $W(\theta)$ should be easy to observe.

In addition to a precise determination of the parameter $\left|J_{s f}\right|$ an experiment on $\mathrm{CeAu}$ could also afford other interesting information. According to the theory $\left({ }^{4}\right)$, the exchange interaction between the conduction electrons and the localized spin in $\mathrm{Ce}^{3+}$ as in $\mathrm{Yb}^{3+}$, is due to a resonant mechanism leading to a negative $J_{s f}$ and subsequently to a Kondo situation. Kondo deviations of $1 / T_{1 \mathrm{~s}}$ with respect to the Korringa law have effectively be shown to exist in $\mathrm{Yb}^{170} \mathrm{Au}$, and similar deviations could be expected to occur in $\mathrm{Ce}^{140} \mathrm{Au}$.

(4) Coqblin, B., Private communication.
3.2.2 $S=1$. - We have computed the integrated perturbation factors both with our theory (assuming relaxation by a fluctuating field $l=1$ ) and with Scherer-Blume theory. It turns out that the difference between the two curves $G_{22}$ is extremely small, while as shown by figure 9 there is $25 \%$ difference between the $G_{44}$ around $1 / \omega T_{1 \mathrm{~s}} \sim 0.25$. Unfortunately $G_{44}$ is often very difficult to observe because of the smallness of the relevant multiplicative coefficient $A_{4}$ in the general expression of $W\left(\mathbf{k}_{1}, \mathbf{k}_{2}, t\right)$.

In practice an effective spin $S=1$ is associated with the irreductible representations $\Gamma_{4}$ and $\Gamma_{5}$ of even electron atoms in a cubic field. But in this case the hyperfine hamiltonian will generally contain not only a magnetic coupling $A \mathbf{I}$.S but also a quadrupole coupling of the form, for a rare earth ion ([33], p. 740731) :

$$
\begin{aligned}
& \mathscr{H}_{\mathrm{Q}}=-\frac{e^{2} Q\left\langle r^{-3}\right\rangle}{I(2 I-1)}\langle L\|\alpha\| L\rangle \times \\
& \times\left[\frac{m}{6} \sum_{q=x, y, z}\left\{3 I_{q}^{2}-I(I+1)\right\}\left\{3 S_{q}^{2}-S(S+1)\right\}\right. \\
& \left.+\frac{3 n}{4} \sum_{\substack{p, q=x, y, z \\
p \neq q}}\left(I_{p} I_{q}+I_{q} I_{p}\right)\left(S_{p} S_{q}+S_{q} S_{p}\right)\right]
\end{aligned}
$$

which should be included in the theory. This will not be trivial since when $m \neq n, \mathbf{F}^{2}$ and $F_{z}$ are not good quantum numbers for $\mathcal{H}_{\mathbf{Q}}$.

4. Perturbation factor in an external field. Evaluation of corrections associated with the off-diagonal part of the hyperfine coupling. - In section 2.6 , we have shown that by working in the representation $m_{I} m_{S}$ and by reducing the hyperfine hamiltonian to its diagonal part $A I_{z} S_{z}$, it was possible to factorize the Liouville matrix of the problem into blocks with dimensions $(2 S+1)^{2} \times(2 S+1)^{2}$. The off diagonal term $(A / 2)\left(I_{+} S_{-}+S_{-} I_{+}\right)$connects these blocks, more precisely it connects submatrices of type $I$ in one block to submatrices of type II in another one. Since the diagonal elements of submatrices II contain the electronic Zeeman energy $g \mu_{\mathrm{B}} H_{0}$ we expect that when $g \mu_{\mathrm{B}} H_{0} \gg A$, the corrections will be of order $A^{2} / g \mu_{\mathrm{B}} H_{0}$ and therefore negligible compared with the diagonal contributions.

Before looking at this in more detail, let us briefly indicate some orders of magnitude. Usually the hyperfine constant $A<\frac{1}{10} \mathrm{~K}$ while standard PAC experiments are performed above $1 \mathrm{~K}$. Condition $g \mu_{\mathrm{B}} H_{0} \gg A$ is satisfied as soon as $H_{0}>$ a few kOe. At $T>1 \mathrm{~K}$ in a few $\mathrm{kOe}$, the electronic spin is still unpolarized : this is the situation which we shall be considering. Appreciable electronic polarization would require much higher fields; relaxation effects in this case were considered in reference [3] with an Abragam-Pound type of approach in the fast relaxation limit, and a simple stochastic approach using 


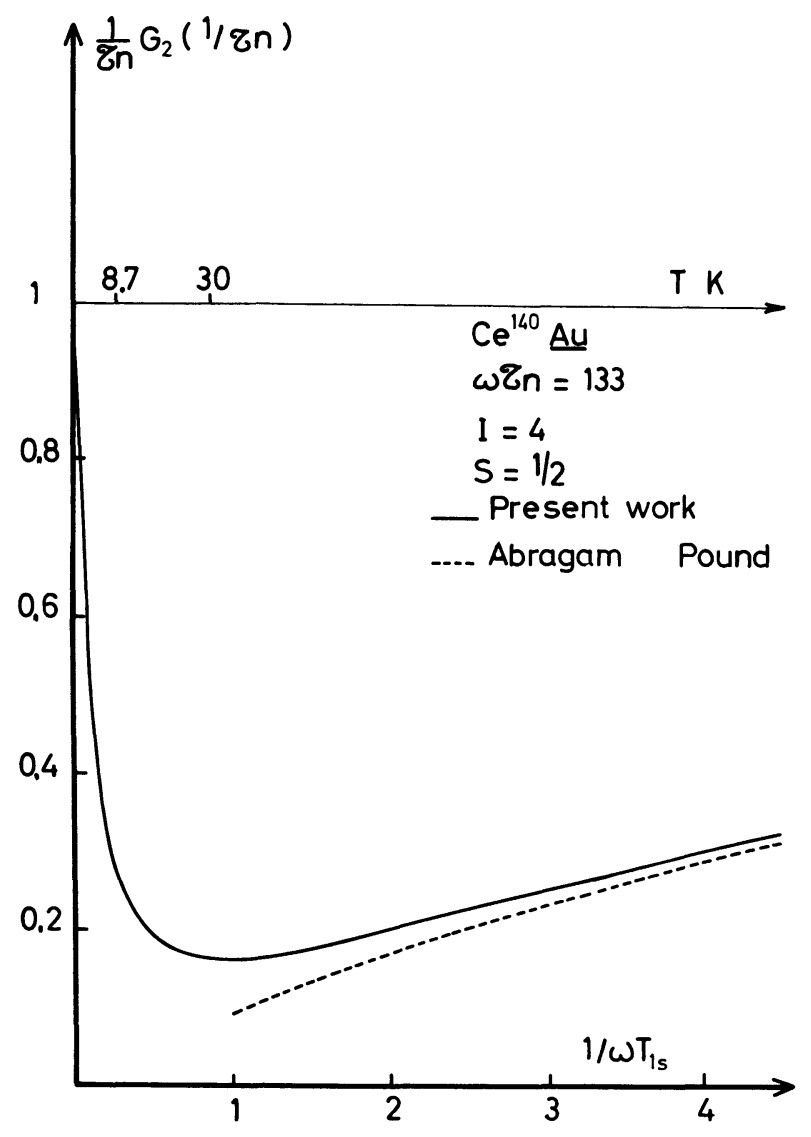

FIG. 8. - Theoretical integral perturbation factor for the $2083 \mathrm{keV}$ state of $\mathrm{Ce}^{1+0} \mathrm{Au}\left(I=4, S=1 / 2, \omega=(A / \hbar)(I+1 / 2),(1) \tau_{\mathrm{n}}=133\right)$. The temperature scale is only tentative (see text).

the truncated hamiltonian $\mathscr{H}_{0}^{\prime}$ for the intermediate region; the conclusions of the present study concerning the smallness of the off-diagonal corrections will clearly also be valid for this problem.

We have computed these corrections in the simplest case, $S=1 / 2, I=1$ by two different methods :

- first, numerical evaluation of the $36 \times 36$ Liouville matrix of the problem,

- second, elaboration of a perturbation treatment adapted to the Liouville formalism.

We will not give the details of the calculations which can be found in reference [34] but only their results, expressed in terms of the quantities $\widetilde{G}_{22}^{00}(p)$, $\tilde{G}_{22}^{22}(p)$ with $p=i \omega$. Since the Liouville matrix did not factorize anyway we have slightly increased the generality of the problem by introducing two electronic relaxation times $T_{1 \mathrm{~s}}$ (longitudinal) and $T_{2 \mathrm{~S}}$ (transverse). Upon setting $\hbar \omega_{0}=g \mu_{\mathrm{B}} H_{0}$, we then obtained that whatever $T_{1 \mathrm{~S}}, T_{2 \mathrm{~S}}$ :

$$
\tilde{G}_{22}^{00}=\frac{1}{i \omega+\frac{\left(3 A^{2} / 2 \hbar^{2} T_{2 \mathrm{~S}}\right)}{\omega_{0}^{2}+\left(1 / T_{2 \mathrm{~S}}\right)^{2}}} ;\left(\frac{A}{\hbar \omega_{0}} \ll 1\right) \text {. }
$$

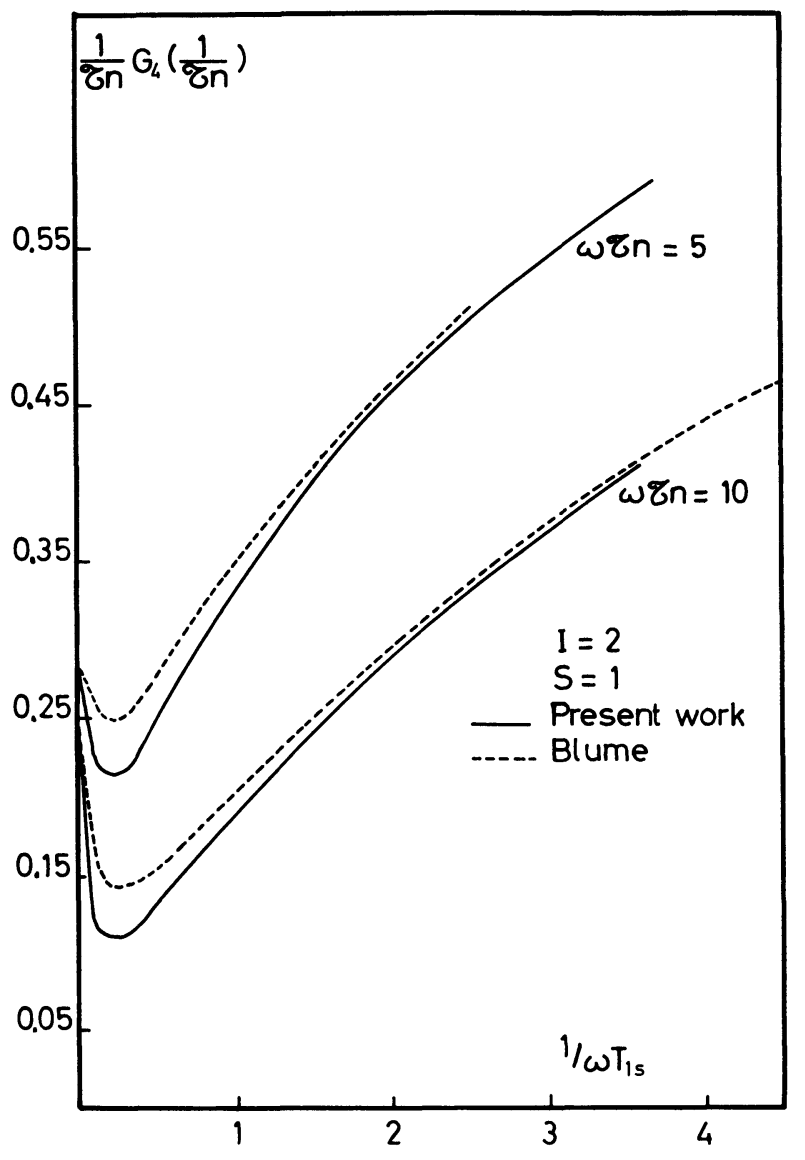

Fig. 9. $-I=2, S=1$. Comparison between the integral perturbation factors $G_{44}\left(1 / \tau_{\mathrm{n}}\right)$ assuming either relaxation by a fluctuating field or Scherer-Blume model with $\lambda \equiv 1 / T_{1}$.

This expression corresponds to a central peak at zero frequency with a broadening (underlined term) entirely due to the off diagonal corrections, whose maximum value is $\frac{3 A^{2}}{4 \hbar^{2} \omega_{0}}$. The associated $G_{22}^{00}(t)$ is purely damped :

$$
G_{22}^{00}=\exp \left(-\mu_{2}^{0} t\right) \quad \text { with } \quad \mu_{2}^{0}=\frac{\left(3 A^{2} / 2 \hbar^{2} T_{2 \mathrm{~S}}\right)}{\omega_{0}^{2}+\left(1 / T_{2 \mathrm{~S}}\right)^{2}} .
$$

In the fast relaxation limit $\left(1 / T_{2 \mathrm{~S}} \gg A\right)$ this result should be identical to that derived in [3] :

$$
\mu_{k}^{q}=\frac{k(k+1)}{2 T_{1 \mathrm{n}}}+q^{2}\left(\frac{1}{T_{2 \mathrm{n}}}-\frac{1}{T_{1 \mathrm{n}}}\right)
$$

in which the nuclear relaxation times $T_{1 \mathrm{n}}, T_{2 \mathrm{n}}$ are given by eq. (14) and (15) of [35]

$$
\frac{1}{T_{1 \mathrm{n}}}=\frac{2}{3} S(S+1) \frac{A^{2}}{\hbar^{2}} \frac{1 / T_{2 \mathrm{~S}}}{\omega_{0}^{2}+\left(1 / T_{2 \mathrm{~S}}\right)^{2}}
$$

$\frac{1}{T_{2 \mathrm{n}}}=\frac{1}{2 T_{1 \mathrm{n}}}+\frac{1}{T_{2 \mathrm{n}}^{\prime}}=\frac{1}{2 T_{1 \mathrm{n}}}+\frac{1}{3} A^{2} S(S+1) T_{1 \mathrm{~S}}$. 
It appears that our expression for $\mu_{2}^{0}$ is indeed in agreement with these previous results.

Concerning $\widetilde{G}_{22}^{22}$ we must distinguish between the fast and slow relaxation cases. The underlined terms in the subsequent expressions represent the offdiagonal corrections.

- Fast electronic relaxation $\left((A / \hbar) T_{1 \mathrm{~s}} \ll 1\right)$ :

$$
\tilde{G}_{22}^{22}=\frac{1}{i\left(\omega-\frac{A^{2} \omega_{0} / 2 \hbar^{2}}{\omega_{0}^{2}+\left(1 / T_{2 \mathrm{~S}}\right)^{2}}\right)+\frac{A^{2} T_{1 \mathrm{~S}}}{\hbar^{2}}+\frac{\left(A^{2} / 2 \hbar^{2} T_{2 \mathrm{~S}}\right)}{\omega_{0}^{2}+\left(1 / T_{2 \mathrm{~S}}\right)^{2}}} ;\left(\frac{A}{\hbar \omega_{0}} \ll 1\right)
$$

The dissipative part in the denominator of this expression is in agreement with eq. (77), (78), (79). It appears that the off diagonal correction is always smaller than the diagonal term $A^{2} T_{1 \mathrm{~S}} / \hbar^{2}$, unless $1 / T_{2 \mathrm{~S}} \gg \omega_{0}$, in which case both terms are comparable and less than $A^{2} / \hbar \omega_{0}$. On the other hand one notices that eq. (80) represents a peak which is shifted from $\omega=0$. This displacement is the nuclear frequency shift associated with the relaxation process [37] i.e. the two underlined terms in the denominator are the real and imaginary parts of :

$\frac{A^{2}}{\hbar^{2}} \int_{0}^{\infty} \frac{\overline{S^{-}(0) S^{+}(-\tau)+S^{+}(-\tau) S^{-}(0)}}{2} \mathrm{~d} \tau$.
The frequency shift is usually neglected in relaxation calculations. It would be difficult to observe since, as soon as the electronic spin is even very slightly polarized, an additional contribution to the frequency appears :

$$
\frac{A\left\langle S_{z}\right\rangle}{\hbar}=\frac{A}{\hbar} \frac{S(S+1) \hbar \omega_{0}}{3 k_{\mathrm{B}} T}=\frac{A}{\hbar} \frac{\hbar \omega_{0}}{4 k_{\mathrm{B}} T}
$$

which will generally overcome the relaxation frequency shift.

- Slow electronic relaxation $\left(\frac{A}{\hbar} T_{1 \mathrm{~s}} \gg 1\right)$ :

$$
\begin{aligned}
\tilde{G}_{22}^{22} \propto \frac{\left(1-i \hbar / 2 A T_{1 \mathrm{~S}}\right)}{i\left(\omega-\frac{A}{\hbar}+\frac{\hbar}{8 A\left(T_{1 \mathrm{~S}}\right)^{2}}-\frac{A^{2} \omega_{0} / 2 \hbar^{2}}{\left(\frac{1}{T_{2 \mathrm{~S}}^{\prime}}\right)^{2}+\omega_{0}^{2}}\right)+\frac{1}{2 T_{1 \mathrm{~S}}}+\frac{\left(A^{2} / 2 T_{2 \mathrm{~S}}^{\prime} \hbar^{2}\right)}{\left(\frac{1}{T_{2 \mathrm{~S}}^{\prime}}\right)^{2}+\omega_{0}^{2}}} & \\
& +\frac{\left(1+i \hbar / 2 A T_{1 \mathrm{~S}}\right)}{i\left(\omega+\frac{A}{\hbar}-\frac{\hbar}{8 A\left(T_{1 \mathrm{~S}}\right)^{2}}-\frac{A^{2} \omega_{0} / 2 \hbar^{2}}{\left(\frac{1}{T_{2 \mathrm{~S}}^{\prime}}\right)^{2}+\omega_{0}^{2}}\right)+\frac{1}{2 T_{1 \mathrm{~S}}}+\frac{\left(A^{2} / 2 T_{2 \mathrm{~S}}^{\prime} \hbar^{2}\right)}{\left(\frac{1}{T_{2 \mathrm{~S}}^{\prime}}\right)^{2}+\omega_{0}^{2}}}\left(\frac{A}{\hbar \omega_{0}} \ll 1\right) .
\end{aligned}
$$

In this equation : $1 / T_{2 \mathrm{~S}}^{\prime}=1 / T_{2 \mathrm{~S}}-1 / 2 T_{1 \mathrm{~S}}$. Notice that it describes a spectrum with two lines at $\omega= \pm A / \hbar$. Here too the off diagonal corrections are small and less than $A^{2} / 4 \hbar \omega_{0}$. If we neglect them, we recover the standard result of simple stochastic theories (see for example Abragam [8] p. 450, eq. (64) with $\Omega=1 / 2 T_{1 \mathrm{~s}}$ and $\left.\delta=A / \hbar\right)$.

The complete evolution of $\operatorname{Re} G_{22}^{22}(\omega)$ from the slow to the fast relaxation limits is represented in figure (10). One starts from a two line spectrum which collapses and is replaced by a single line, in agreement with standard descriptions. On the other hand the variation of $G_{22}^{22}(\omega)$ as a function of the external field in the slow relaxation limit is represented in figure 11 .

If we now gather the preceding results eq. (76), (80), (83) it appears that as long as $T_{1 \mathrm{~S}}$ and $T_{2 \mathrm{~S}}$ are comparable, the off-diagonal corrections are generally negli-

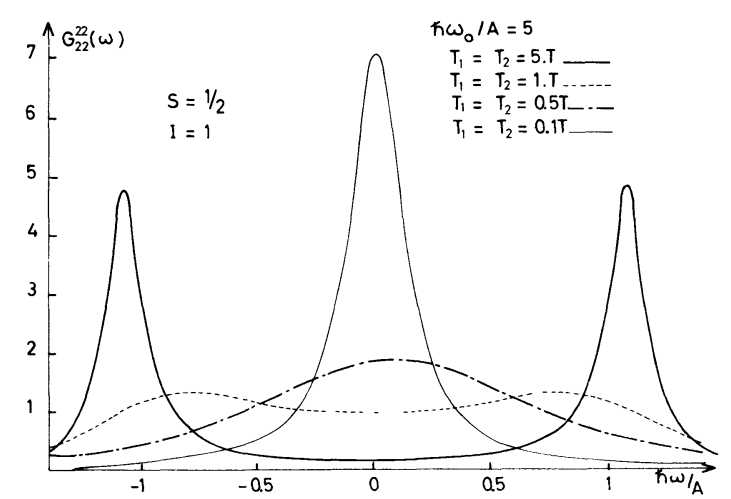

FIG. 10. $-I=1, S=1 / 2$. Fourier transform $G_{22}^{22}(\omega)$ of the perturbation factor in high field for various values of $T_{1 \mathrm{~S}}$ (computer calculation).

gible compared to the contribution of the truncated hamiltonian (the only exception being the case of 


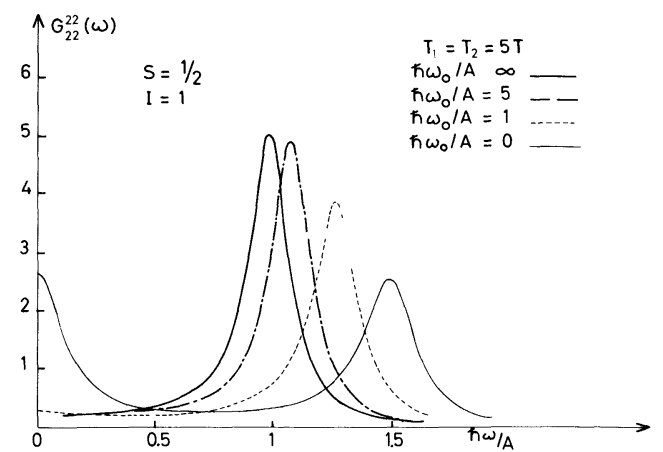

FIG. 11. $-I=1, S=1 / 2$. Fourier transform $G_{22}^{22}(\omega)$ of the perturbation factor in the slow relaxation limit for various values of the field (computer calculation).

extremely fast relaxation, $1 / T_{2 \mathrm{~S}} \gg \hbar \omega_{0}$, for which all contributions are very small). Since these corrections represent the deviations with respect to the simple stochastic model, this shows the interest of this model when $g \mu_{\mathrm{B}} H_{0} \gg A$.

5. Conclusion. - In conclusion we have extended Hirst's relaxation theory to the case of perturbed angular correlations.

- In the absence of a magnetic field, the formalism of irreducible tensor operators, which is well adapted to the spherical symmetry of the problem, has made it possible to derive simple equations of motion for the electronuclear density matrix - the parameter $\lambda_{k_{s}}$ which comes into play in these equations being clearly related to the fluctuating microscopic hamiltonian responsible for the electronic relaxation. The SchererBlume theory has been shown to be a particular case of our general treatment, corresponding to the situation : $\lambda_{k_{s}}=\lambda$ for all $k_{s}$. We have computed numerical values of the perturbation factors for a number of cases. As concerns the experimental checking of our theory, DPAC experiments do not seem to be feasible at the present time, because of the unsufficient time resolution of the electronics. On the contrary IPAC appear to be quite suitable and we discuss three possible experiments on ${ }_{70} \mathrm{Yb}^{172}$ and ${ }_{58} \mathrm{Ce}^{140}$ at low temperatures.

- On the other hand, in the presence of an electronic Zeeman effect which is large compared with the hyperfine splitting, we have demonstrated that our theory reduces to standard diagonal stochastic treatments. For this, using the Liouville formalism, we have evaluated the non-adiabatic corrections associated with the off-diagonal part of the hyperfine hamiltonian; these corrections have been found to be smaller than the adiabatic contributions by factors of the order $A / \hbar \omega_{0}$ or $\left(A / \hbar \omega_{0}\right)^{2}$.

\section{APPENDIX I}

Abragam-Pound limit. - In this Appendix we wish to give a new comparison of our approach with that of Scherer and Blume, and to show that in the appropriate limit our theory reduces to the result of Abragam and Pound. For this we shall use the uncoupled basis tensor operators

$$
\begin{array}{r}
{ }_{k_{I} k_{S}}^{U_{K}^{Q}}=\sum_{q_{I} q_{S}}\left\langle k_{I} q_{I} k_{S} q_{S} \mid K Q\right\rangle T_{k_{I}}^{q_{I}}(I) T_{k_{S}}^{q_{S}}(S) \\
\left(\left|k_{I}-k_{S}\right| \leqslant K \leqslant\left|k_{I}+k_{S}\right| ;\right. \\
\left.k_{I}=0 \ldots 2 I ; \quad k_{S}=0 \ldots 2 S\right) .
\end{array}
$$

In terms of this operator :

$$
T_{\mathrm{K}}^{Q}(I) T_{0}^{0}(S)={ }^{\mathrm{Ko}} U_{\mathrm{K}}^{Q} .
$$

Since $T_{0}^{0}(S)=1 / \sqrt{2 S+1}$ we may use eq. (19) to derive a new expression of the perturbation factor

$$
\begin{aligned}
& G_{K^{\prime} \mathbf{K}}^{Q^{\prime}}\left(t, t^{\prime}\right)=\operatorname{Trace}_{I S}\left\{\left({ }^{K o} U_{\mathbf{K}}^{Q+}\right) \Lambda\left(t, t^{\prime}\right) \times\right. \\
& \left.\times\left({ }^{K^{\prime} O} U_{\bar{K}^{\prime}}^{Q^{\prime}}\right) \Lambda^{+}\left(t, t^{\prime}\right)\right\} \\
& =\operatorname{Trace}_{I S}\left\{{ }^{\boldsymbol{K}^{\prime} O} U_{\mathbb{K}^{\prime}}^{Q^{\prime}}(O){ }^{K o} U_{\widetilde{K}}^{Q+}\left(t, t^{\prime}\right)\right\} \text {. }
\end{aligned}
$$

We want to compute $G$ in the presence of the hyperfine coupling $\mathfrak{H}_{0}=A \mathbf{I}$.S and of electronic relaxation. We have already shown in the main text that $G_{K^{\prime}, K}^{Q^{\prime}}$ is non zero only if $K^{\prime}=K, Q^{\prime}=Q$ and that it does not depend on $Q$ :

$$
G_{\overline{K K}}^{Q Q}(t)=G_{K K}^{00}(t)=G_{K}(t) .
$$

We can obtain it by writing the equation of evolution of the ${ }^{k k^{\prime}} U_{\mathrm{K}}^{Q}$ in the Heisenberg representation. The electronic relaxation term is very simple and we have that :

$$
\frac{\mathrm{d}}{\mathrm{d} t}\left({ }^{k k^{\prime}} U_{\widetilde{K}}^{Q}\right)=\frac{i}{\hbar}\left[\mathcal{H}_{0},\left({ }^{k k^{\prime}} U_{\bar{K}}^{Q}\right)\right]-\lambda_{k^{\prime}}\left({ }^{k k^{\prime}} U_{\mathbf{K}}^{\varrho}\right)
$$

$K$ and $Q$ are good quantum numbers for $\mathcal{H}_{0}=A \mathbf{I} . \mathbf{S}$ (see main text) and eq. (A.4) takes the form :

$$
\begin{aligned}
& \frac{\mathrm{d}}{\mathrm{d} t}\left({ }^{k k^{\prime}} U_{\bar{K}}^{Q}\right)=i \sum_{k_{1} k_{1}^{\prime}} \alpha_{K}\left(k k^{\prime} \mid k_{1} k_{1}^{\prime}\right)\left({ }^{k_{1} k_{1}^{\prime}} U_{K}^{Q}\right)- \\
& -\lambda_{k^{\prime}}\left(k^{\prime} U_{K}^{Q}\right)
\end{aligned}
$$

where the coefficient $\alpha_{K}$ have the following properties $\alpha_{K}(00 \mid 00)=0$

$\alpha_{K}\left(00 \mid k k^{\prime}\right)=\alpha_{K}\left(k k^{\prime} \mid 00\right)$ are zero unless $k^{\prime}=1$

Let us write

$$
{ }^{k k^{\prime}} U_{\mathbb{K}}^{Q}=\left\langle k k^{\prime}|U| K Q\right\rangle
$$


and define a column vector $U=$\begin{tabular}{|c|}
\hline$X$ \\
\hline$Y$ \\
\hline
\end{tabular} with $X={ }^{K o} U_{\mathbf{K}}^{Q}$ and

$$
Y=\left[\begin{array}{c}
k^{-1} U_{\mathbf{K}}^{Q} \\
\cdots \\
k^{2} U_{\mathbf{K}}^{Q} \\
\ldots
\end{array}\right]|K-1| \leqslant k \leqslant|K+1|
$$

Eq. (A.5) becomes, in matrix form :

$$
\frac{\mathrm{d} U}{\mathrm{~d} t}=(M+R) U
$$

where the matrices $M$ and $R$ have the form :
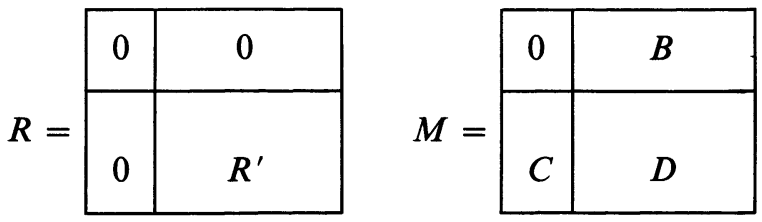

i.e. the first line and first column of $R$ are zero and the remaining part $R^{\prime}$ is a diagonal matrix with elements

$$
\begin{aligned}
& -\lambda_{1}(|K-1| \leqslant k \leqslant|K+1|) \\
& -\lambda_{2}(|K-2| \leqslant k \leqslant|K+2|) \ldots
\end{aligned}
$$

(let us recall that $\lambda_{0}=0$, because of the constancy of the sum of the electronic populations in the absence of nuclear spin).

In $M$, the only non zero elements of the line and column vectors $B$ and $C$ are the $i \alpha_{K}(00 \mid k 1)$ and $i \alpha_{K}(k 1 \mid 00)$.

We may solve eq. (A.9) by Laplace transformation :

$$
\tilde{U}(p)=\frac{\tilde{U}(0)}{p J-R-M}
$$

in which $J$ is the unit matrix. Let us define the inverse matrix of $p J-R-M$ as

$$
\left(\begin{array}{l|l}
A^{\prime} & B^{\prime} \\
\hline C^{\prime} & D^{\prime}
\end{array}\right)=\left(\begin{array}{c|c}
p & -B \\
\hline-C & p J-R^{\prime}-D
\end{array}\right)^{-1} .
$$

Taking account of (A.11), eq. (A.9) becomes :

$$
\begin{aligned}
& \tilde{X}(p)=A^{\prime} \tilde{X}(0)+B^{\prime} \tilde{Y}(0) \\
& \tilde{Y}(p)=C^{\prime} \tilde{X}(0)+D^{\prime} \tilde{Y}(0) .
\end{aligned}
$$

On the other hand, by definition of $\tilde{G}_{\overline{K K}}^{Q Q}(p)$, this quantity characterizes the transformation of $X(p)$ into itself. Consequently it is equal to $A^{\prime}$. This last matrix can be computed with the help of Aitken's theorem [36], giving the result :

$$
\begin{aligned}
\tilde{G}_{K K}^{Q Q}(p)= & \tilde{G}_{K}(p)= \\
& =\left[p-B \frac{1}{p J-D-R^{\prime}} C\right]^{-1} .
\end{aligned}
$$

\section{Comparison with Scherer and Blume}

In the absence of relaxation $\left(R^{\prime}=0\right)$

$$
\tilde{G}_{K}^{0}(p)=\left[p-B \frac{1}{p J-D} C\right]^{-1} .
$$

If $R^{\prime}$ is proportional to the unit matrix

$$
R^{\prime}=-\lambda J \text { i.e. } \lambda_{1}=\lambda_{2}=\lambda_{3}=\lambda_{2 \mathrm{~s}}=\lambda
$$

it is easy to check that :

$$
\left[\tilde{G}_{\mathbf{K}}(p)\right]^{-1}=\left[\tilde{G}_{\mathbf{K}}^{0}(p+\lambda)\right]^{-1}-\lambda
$$

or

$$
\tilde{G}_{\boldsymbol{K}}(p)=\frac{\tilde{G}_{\boldsymbol{K}}^{0}(p+\lambda)}{1-\lambda \tilde{G}_{\mathbf{K}}^{0}(p+\lambda)}
$$

i.e. we recover Scherer and Blume's result. Condition (A.15) is identical to eq. (50) established in the main text.

\section{Abragam and Pound limit}

Assume that the electronic relaxation is very fast. Then $\lambda_{1}, \lambda_{2}, \ldots$ are very large and :

$$
\frac{1}{p J-D-R^{\prime}} \simeq-\frac{1}{R^{\prime}}
$$

- $\left(1 / R^{\prime}\right)$ is a diagonal matrix with elements $1 / \lambda_{1}$, $1 / \lambda_{2}, \ldots$ On the other hand we have said that the only non zero matrix elements of $B$ and $C$ are

$$
i \alpha(00 \mid k, 1) \text { and } i \alpha(k, 1 \mid 00) \text {. }
$$

Consequently :

$$
\begin{aligned}
\tilde{G}_{K}(p) & =\frac{1}{p-\frac{1}{\lambda_{1}} \sum_{k} \alpha_{K}(00 \mid k 1) \alpha_{K}(k 1 \mid 00)} \\
& =\frac{1}{p+\mu_{K}} .
\end{aligned}
$$

Notice that $1 / \lambda_{1}=T_{1 \mathrm{~s}}$. There remains to compute $\sum_{k} \alpha_{K}(00 \mid k, 1) \quad \alpha_{K}(k, 1 \mid 00)$. Instead of doing this directly, we remark that eq. (A.19) does not depend on $\lambda_{2}, \lambda_{3}, \ldots, \lambda_{2 s}$. Therefore it remains valid if we replace $\lambda_{2}, \lambda_{3}, \ldots, \lambda_{2 S}$ in $\left(R^{\prime}\right)$ by $\lambda_{1}$. We then recover the Scherer and Blume model with $\lambda=\lambda_{1}$. On the other hand Blume has shown that for large $\lambda$ his $\widetilde{G}_{K}(p)$ takes the form $1 /\left(p+\mu_{K}\right)$ with (in his notation)

$$
\mu_{\mathbf{K}}=\frac{1}{\lambda} \sum_{v} C_{\mathbf{K}}^{v} \omega_{v}^{2}
$$

Consequently

$$
-\sum_{k} \alpha_{K}(00 \mid k 1) \alpha_{K}(k 1 \mid 00)=\sum_{v} C_{K}^{v} \omega_{v}^{2}
$$


where

$$
\begin{aligned}
C_{\boldsymbol{K}}^{v} & =C_{\boldsymbol{K}}^{F F^{\prime}}= \\
& =\frac{(2 F+1)\left(2 F^{\prime}+1\right)}{(2 S+1)}\left\{\begin{array}{ccc}
I & F & S \\
F^{\prime} & I & K
\end{array}\right\}^{2}
\end{aligned}
$$

and

$$
\begin{aligned}
\hbar \omega_{v}=E_{F}- & E_{F^{\prime}}= \\
& =\frac{A}{2}\left[F(F+1)-F^{\prime}\left(F^{\prime}+1\right)\right] .
\end{aligned}
$$

Evaluation of $\sum_{v} C_{K}^{v} \omega_{v}^{2}$ is achieved by using summa- tion rules over $6 j$ symbols (Edmonds [20] eq. (6.2.9) and (6.2.12)) and we finally get :

$$
\mu_{K}=\frac{A^{2} K(K+1) S(S+1)}{3} T_{1 \mathrm{~s}}
$$

i.e. the Abragam and Pound result.

Notice that, despite the fact that for general $S$ our theory and the Scherer-Blume theory are not equivalent, in the limit of short relaxation times, they both reduce to the Abragam and Pound limit provided that one takes $\lambda=1 / T_{1 \mathrm{~s}}$.

\section{APPENDIX II}

Application of tensor operators to the computation of the Mössbauer lineshape. - We have also used the tensor operator method of the present paper to compute the Mössbauer lineshape of $\mathrm{Yb}^{170} \mathrm{Au}$ at temperatures $T>1 \mathrm{~K}$ where population effects can be neglected [15]. It turns out that in the case of cubic symmetry $\left(A_{\|}=A_{\perp}, W_{\|}=W_{\perp}, \beta=0\right)$ and in the absence of external field $(\alpha=0)$, eq. (77) of reference [5] can be replaced by the equivalent but much simpler expression :

$$
F(p)=5 \frac{\left(p-\frac{i A}{2 \hbar}+\frac{1}{T_{1 \mathrm{~s}}}\right)}{p^{2}+p\left(\frac{1}{T_{1 \mathrm{~s}}}-\frac{i A}{2 \hbar}\right)+\frac{3 A^{2}}{2 \hbar^{2}}}
$$

(with $\left.p=\frac{\Gamma}{2}-i \omega\right)$. Details of the calculations are given in chapitre 4 of reference [34].

\section{References}

[1] Abragam, A., Pound, R. V., Phys. Rev. 92 (1953) 943.

[2] Gabriel, H., Phys. Rev. 181 (1969) 509.

[3] Spanjaard, D., Hartmann-Boutron, F., J. Physique 30 (1969) 975 .

[4] HIRST, L. L., J. Phys. \& Chem. Solids 31 (1970) 655.

[5] GonZalez-Jimenez, F., Imbert, P., Hartmann-Boutron, F., Phys. Rev. B 9 (1974) 95.

[6] Hartmann-Boutron, F., Spanjaard, D., J. Physique 36 (1975) 307

[7] StefFen, R. M., Frauenfelder, H., in Perturbed Angular correlations (Karlsson, Matthias, Siegbahn ed. North Holland) 1964, p. 2.

[8] Abragam, A., The Principles of Nuclear Magnetism (Oxford University Press) 1961.

[9] SCHERER, C., Nucl. Phys. A 157 (1970) 81.

[10] Blume, M., Nucl. Phys. A 167 (1971) 81.

Similar results are derived by a different method in : DATTAGupta, S., Blume, M., Phys. Rev. B 10 (1974) 4540.

[11] Clauser, M. J., Blume, M., Phys. Rev. B 3 (1971) 583.

[12] A similar analysis was already used in a different context by Abragam, A., Phys. Rev. 98 (1955) 1729.

[13] Hartmann-Boutron, F., Spanjaard, D., J. Physique 33 (1972) 285.

[14] Spanjaard, D., Hartmann-Boutron, F., J. Physique 33 (1972) 565.

[15] a) Hartmann-Boutron, F., Phys. Rev. B 10 (1974) 2113.

b) GonZalez-Jimenez, F., Hartmann-Boutron, F., Imber T, P., Phys. Rev. B 10 (1974) 2122.

[16] Gonzalez-Jimenez, F., Cornut, B., Coqblin, B., Phys. Rev. B 11 (1975) 4674

[17] Chopin, C., Hartmann-Boutron, F., Spanjaard, D., $J$. Physique colloq. 35 (1974), C6-433.

[18] The factor $1 /(2 S+1)$ has been omitted in the formulas of ref. [7].

[19] Ducloy, M., Dumont, M., J. Physique 31 (1970) 419.
[20] Edmonds, A. R., Angular Momentum in Quantum Mechanics (Princeton University Press) 1957.

[21] Ref. [7], eq. (109), taking account of the remark [18].

[22] Gabriel, H., Phys. Lett. 32A (1970) 202.

[23] a) Gunther, C., Blumberg, H., Engels, W., Strube, G., Voos, J., LIEDeR, R. M., Luig, H., BOdenstedt, E., Nucl. Phys. 61 (1965) 65.

b) Blumberg, H., Speidel, K. H., Schlenz, H., Weigt, P., Hübel, H., Göttel, P., WaGNER, H. F., Bodenstedt, E., Nucl. Phys. A 90 (1967) 65.

[24] Wimmer, L., Z. Naturforsch 28a (1973) 226.

[25] Stautberg, M. M., Shera, E. B., Casper, K. J., Phys. Rev. 130 (1963) 1901.

[26] Günther, C., Engels, W., Bodenstedt, E., Phys. Lett. 10 (1964) 77.

[27] Wagner, H. F., Lange, J., Z. Phys. 238 (1970) 35.

[28] Hirst, L. L., Stöhr, J., Shenoy, G. K., Kalvius, G. M., Phys. Rev. Lett. 33 (1974) 198.

[29] Flouquet, J., Annls de Phys. 8 (1974) 28.

[30] Körner, H. J., Gerdau, E., Günther, C., Averbach, K., Mielken, G., Strube, G., Bodenstedt, E., Z. Phys. 173 (1963) 203.

[31] Levy, R. M., Shirley, D. A., Phys. Rev. 140B (1965) 811.

[32] Caillau, M., Thèse de troisième cycle Orsay (1969).

[33] Abragam, A., Bleaney, B., Electron paramagnetic resonance of transition ions (Clarendon Press, Oxford) 1970.

[34] Chopin, C., Thèse de troisième cycle Orsay (1975).

[35] Spanjaard, D., Hartmann-Boutron, F., J. Phys. F. (G. B.) 3 (1973) 1178.

[36] See the Appendix of ref. [14]. Also : Artken, J. Determinants and Matrices (Wiley), p. 148.

[37] Hartmann-Boutron, F., Thèse de Doctorat d'Etat, Paris (1963) (unpublished). Appendix B.

[38] Ducloy, M., Thèse de troisième cycle, Paris (1968). Appendix II. See also eq. (1.48) and (1.49) of reference [19] 